\title{
Structural, Spectroscopic (FT-IR, Raman, NMR and UV-Vis.) and Theoretical Investigations of Cyclopentolate
}

\author{
Tuba Özdemir Öge ${ }^{1 *} \odot$, Halil Gökçe² \\ ${ }^{1}$ Vocational School of Health Services, Bartın University, 74100 Bartın, Turkey \\ ${ }^{2}$ Vocational School of Health Services, Giresun University, 28200 Giresun, Turkey \\ Geliş / Received: 11/10/2019, Kabul / Accepted: 18/03/2020
}

\begin{abstract}
In this study, the structural and spectroscopic characterizations (FT-IR, Laser-Raman, ${ }^{1} \mathrm{H}$ and ${ }^{13} \mathrm{C}$ NMR and UV-vis spectra) of cyclopentolate were performed using experimental and theoretical methods. Four conformational structures and optimized molecular geometries of the molecule were examined. The vibrational wavenumbers, proton and carbon-13 NMR chemical shifts, UV-Vis. parameters were theoretically obtained for the most stable form of the molecule and the HOMO-LUMO analyses were performed with DFT/B3LYP method at aug-cc-pVDZ basis set. The weak intra-molecular hydrogen bond interaction in O45-H46...N23 group was determined. The ${ }^{1} \mathrm{H}$ and ${ }^{13} \mathrm{C}$ NMR chemical shifts were experimentally and theoretically investigated. The assignments of theoretical vibrational modes were obtained using VEDA 4 software in terms of potential energy distribution (PED). The theoretically and experimentally obtained data were found to be consistent with each other.
\end{abstract}

Keywords: Cyclopentolate, Vibrational spectroscopy, UV-Vis. spectral parameters, HOMO-LUMO analyses, NMR chemical shifts

\section{Siklopentolat Molekülünün Yapısal, Spektroskopik (FT-IR, Raman, NMR ve UV-Vis.) ve Teorik Olarak İncelenmesi}

\section{Özet}

Bu çalışmada siklopentolat molekülünün yapısal ve spektroskopik karakterizasyonları (FT-IR, Laser-Raman, ${ }^{1} \mathrm{H}$ ve ${ }^{13} \mathrm{C}$ NMR ve görünür UV spektrumları) deneysel ve teorik yöntemler kullanılarak incelendi. Molekülün dört adet konformasyon yapısı ve optimize edilmiş moleküler geometrileri incelendi. En kararlı form için molekülün titreşimsel dalga-boyları, ${ }^{1} \mathrm{H}$ ve ${ }^{13} \mathrm{C}$ NMR kimyasal kaymaları, görünür UV (UV-Vis) parametreleri teorik olarak elde edildi ve HOMO-LUMO analizleri DFT/B3LYP yöntemi ile aug-cc-pVDZ temel seti kullanılarak gerçekleştirildi. O45-H46...N23 grubundaki zayıf molekül-içi hidrojen bağ etkileşimi belirlendi. Teorik titreşim mod atamaları VEDA 4 yazılımı kullanılarak potansiyel enerji dağılımı (PED) cinsinden elde edildi. Teorik ve deneysel yöntemlerle elde edilen verilerin birbirleri ile tutarlı oldukları belirlendi.

Anahtar Kelimeler: Siklopentolat, Titreşim spektroskopisi, Görünür UV. Spektrum parametreleri, HOMOLUMO analizleri, NMR kimyasal kaymaları

\footnotetext{
*Corresponding Author: tozdemir@bartin.edu.tr
} 


\section{Introduction}

Cycloplegia is the temporary paralysis of siliceous muscles that provide accommodation with the aid of various drops. Cycloplegia agents include atropine, homatropine, cyclopentolate, scopolamine and tropicamide (Özer, 2005). Cycloplegic drugs, also referred to as anticholinergics, block the muscarinic action of acetylcholine. This action inhibits cholinergic stimulation of the iris sphincter and ciliary muscle, which results in mydriasis and cycloplegia (Mindel, 1994). Drugs acting like acetylcholine at the receptor such as pilocarpine are called muscarinic agonists; and drugs blocking the action of acetylcholine at the receptor such as atropine, homatropine and cyclopentolate are called muscarinic antagonists (Vale and Cox, 1979). Cyclopentolate hydrochloride is a cycloplegic and mydriatic agent and an ester of a substituted benzeneacetic acid, having, $\mathrm{N}$, $\mathrm{N}$-dimethylaminoethanol as the alcohol moiety (Roy and Guillory, 1995). Cyclopentolate hydrochloride's (CPH) IUPAC name is 2-(dimethylamino)-ethyl-1hydroxy- $\alpha$-phenylcyclopentaneacetate hydrochloride, molecular weight is 327.85 $\mathrm{g} / \mathrm{mol}$ and molecular formula is $\mathrm{C}_{17} \mathrm{H}_{25} \mathrm{NO}_{3} . \mathrm{HCl}$ (Frag et al, 2011). 5 Cycloplegic drops have been applied to observe the effects of the drugs in various studies. Hung et al, evaluated the changes of intraocular pressure and refractive status among children (Hung et al, 2015). Farhood assessed the cycloplegic refraction among children. Their study aimed to evaluate the safety and efficacy of two cycloplegic regimens among hyperopic children (Farhood, 2012). Atropine 1\% (regimen I) and cyclopentolate $1 \%$ (regimen II) eye drops were evaluated on fifty children. $\mathrm{Li}$ et al. investigated the effects of intravitreal injection of cyclopentolate on form deprivation myopia in guinea pigs (Zhou et al,
2015). Enantiomers, also known as optical isomers, represent each of the two molecules that are mirror images of each other. Different analytical techniques have been suggested in the literature to separate enantiomers and perform enantioselective analyses of chiral compounds (Izake, 2007; Mericko et al, 2007).

The aim of this study is to investigate the structural and spectroscopic analyses of cyclopentolate molecule used in eye treatments. Conformational analysis was performed, and four conformation energy values were calculated. The four conformational structures of the cyclopentolate molecule involve in-plane, out-of-plane $\mathrm{OH}$ bonds, and inward and outward oriented $\mathrm{C}=\mathrm{O}$ bonds (Figure 1 ). The results of all spectroscopic analyses are given for conformation $I$ in the study. The vibrational wavenumbers (FT-IR and LaserRaman), UV analysis and ${ }^{1} \mathrm{H}$ and ${ }^{13} \mathrm{C}$ NMR chemical shifts of cyclopentolate were experimentally and theoretically studied. The recorded experimental data (FT-IR, LaserRaman, UV, NMR chemical shifts) were supported with parameters computed using theoretical methods with the DFT/B3LYP/aug-cc-pVDZ level. 

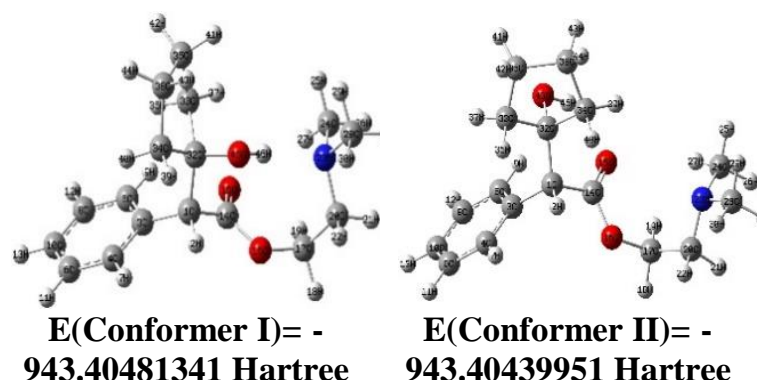

943.40481341 Hartree

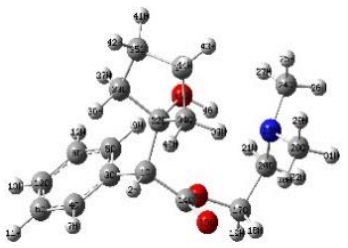

E $($ Conformer III $)=$ 943.40431503 Hartree
E $($ Conformer II $)=$ 943.40439951 Hartree

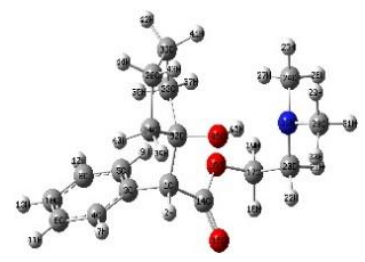

$\mathrm{E}($ Conformer IV $)=$ 943.40317790 Hartree

Figure 1. The optimized four molecular conformational forms in the lowest energy for cyclopentolate

\section{Experimental Analyses}

Cyclopentolate was purchased from SigmaAldrich Corporation. The FT-IR spectrum of cyclopentolate was collected in the region of $400-4000 \mathrm{~cm}^{-1}$ at room temperature on a Perkin-Elmer Spectrum Two FT-IR Spectrometer with standard resolution of 0.5 $\mathrm{cm}^{-1}$ in the transmission mode in the solid phase of the sample. The samples were compressed into self-supporting pellets and introduced into an IR cell equipped with $\mathrm{KBr}$ window.

The Laser-Raman spectrum was recorded on a Renishaw Invia Raman Microscope Spectrophotometer in $100-4000 \mathrm{~cm}^{-1}$ region in the solid phase of the sample. The excitation line width, scan number and resolution of the diode laser were $785 \mathrm{~nm}, 100$ and $1 \mathrm{~cm}^{-1}$, respectively.

The ${ }^{1} \mathrm{H}$ and ${ }^{13} \mathrm{C}$ NMR chemical shifts of the sample dissolved in DMSO-d6 were obtained by Bruker Biospin-Avance III $400 \mathrm{MHz}$ NMR spectrometer at the temperature of $295 \mathrm{~K}$. The chemical shifts were reported at a frequency

of $400 \mathrm{MHz}$ at ppm level relative to tetramethylsilane (TMS).

The ultraviolet visible spectrum of cyclopentolate dissolved in methanol was recorded using a UV-6100 Double Beam Spectrophotometer in the $200-400 \mathrm{~nm}$ region at room temperature. The spectral bandwidth is $2 \mathrm{~nm}$.

\section{Computational Procedures}

The optimized molecular geometries, vibrational wavenumbers, proton and carbon13 NMR chemical shifts, UV-Vis. electronic absorption parameters and HOMO-LUMO analyses of the title molecule were computed using B3LYP (Becke's three parameter exact exchange-functional (B3) combined with gradient-corrected correlational functional of Lee, Yang, Parr (LYP)) functional in density functional theory (DFT) with aug-cc-pVDZ basis set (Becke, 1993; Lee et al, 1988). The molecular geometric parameters, spectral properties and other molecular properties were performed using Gaussian 09W program package on a computing system (Frisch et al, 2009). GaussView5 program was used for visualization of the computed structural, spectroscopic and electronic properties (Dennington et al, 2019).

The calculated vibrational frequencies were scaled by 0.970 for the B3LYP/aug-cc-pVDZ level (Johnson, 2016). The assignments of vibrational wavenumbers and evaluation of potential energy distribution (PED) were performed using VEDA 4 program (Jamr'oz, 2004). The molecule was optimized in DMSO and methanol using conductor polarizable continuum model (CPCM) (Miertus et al, 1981) at the mentioned computational level to obtain ${ }^{1} \mathrm{H}$ and ${ }^{13} \mathrm{C}$ NMR isotropic chemical shifts and UV-Vis. parameters of cyclopentolate molecule. Then, ${ }^{1} \mathrm{H}$ and ${ }^{13} \mathrm{C}$ NMR chemical shifts of the title molecule 
were studied using the gauge invariant atomic orbital (GIAO) approach (Ditchfield, 1974; Wolinski et al, 1990, London, 1937). The UVVis. spectroscopic analysis for the molecule was theoretically performed using the TDDFT method (Runge and Gross, 1984). The HOMO and LUMO analyses were simulated at the aforementioned computational level.

\section{Results and Discussions}

\subsection{Geometric Structure}

The optimized molecular structures and computed molecular geometric parameters (bond lengths and angles) with the B3LYP/aug-cc-pVDZ level of cyclopentolate molecule are given in Figure 1 and Table 1, respectively. The molecular formula of cyclopentolate is $\mathrm{C}_{17} \mathrm{H}_{25} \mathrm{NO}_{3}$ and its chemical name is 2-(Dimethylamino)ethyl(2R)-(1hydroxycyclopentyl)(phenyl)acetate. 


\begin{tabular}{|c|c|c|c|c|c|c|}
\hline Bond lengths $(\AA)$ & Literature & Calc. & Bond angles $\left(^{\circ}\right)$ & Calc. & Bond angles $\left({ }^{\circ}\right)$ & Calc. \\
\hline $\mathrm{C} 1-\mathrm{H} 2$ & - & 1.095 & $\mathrm{H} 2-\mathrm{C} 1-\mathrm{C} 3$ & 107.9 & N23-C24-H25 & 109.2 \\
\hline C1-C3 & - & 1.523 & $\mathrm{H} 2-\mathrm{C} 1-\mathrm{C} 14$ & 106.6 & N23-C24-H26 & 112.0 \\
\hline C1-C14 & - & 1.521 & $\mathrm{H} 2-\mathrm{C} 1-\mathrm{C} 32$ & 104.3 & N23-C24-H27 & 110.5 \\
\hline C1-C32 & - & 1.580 & C3-C1-C14 & 113.0 & $\mathrm{H} 25-\mathrm{C} 24-\mathrm{H} 26$ & 108.2 \\
\hline C3-C4 & \multirow{6}{*}{$\begin{array}{l}\text { d } 1.360-1.410 \\
\text { e } 1.3859-1.4151 \\
\text { e } 1.3920-1.3990\end{array}$} & 1.405 & C3-C1-C32 & 116.2 & $\mathrm{H} 25-\mathrm{C} 24-\mathrm{H} 27$ & 107.9 \\
\hline C3-C5 & & 1.405 & C14-C1-C32 & 108.1 & H26-C24-H27 & 109.1 \\
\hline C4-C6 & & 1.397 & C1-C3-C4 & 118.5 & N23-C28-H29 & 109.5 \\
\hline $\mathrm{C} 5-\mathrm{C} 8$ & & 1.398 & C1-C3-C5 & 123.3 & N23-C28-H30 & 109.7 \\
\hline C6-C10 & & 1.398 & C4-C3-C5 & 118.2 & N23-C28-H31 & 112.4 \\
\hline $\mathrm{C} 8-\mathrm{C} 10$ & & 1.398 & C3-C4-C6 & 121.3 & H29-C28-H30 & 108.1 \\
\hline C4-H7 & \multirow{5}{*}{$\begin{array}{l}\text { e } 1.0756-1.1015 \\
\text { e } 1.0831-1.1010\end{array}$} & 1.091 & C3-C4-H7 & 119.4 & H29-C28-H31 & 108.3 \\
\hline C5-H9 & & 1.087 & C6-C4-H7 & 119.4 & H30-C28-H31 & 108.7 \\
\hline C6-H11 & & 1.091 & $\mathrm{C} 3-\mathrm{C} 5-\mathrm{C} 8$ & 120.7 & C1-C32-C33 & 116.6 \\
\hline C8-H12 & & 1.091 & C3-C5-H9 & 119.6 & C1-C32-C34 & 112.6 \\
\hline C10-H13 & & 1.091 & C8-C5-H9 & 119.7 & C1-C32-O45 & 108.5 \\
\hline C14-O15 & - & 1.215 & C4-C6-C10 & 120.0 & C33-C32-C34 & 102.6 \\
\hline C14-O16 & - & 1.357 & C4-C6-H11 & 119.8 & C33-C32-O45 & 110.5 \\
\hline O16-C17 & - & 1.443 & C10-C6-H11 & 120.2 & C34-C32-O45 & 105.4 \\
\hline C17-H18 & - & 1.096 & $\mathrm{C} 5-\mathrm{C} 8-\mathrm{C} 10$ & 120.6 & C32-C33-C35 & 104.3 \\
\hline C17-H19 & - & 1.094 & C5-C8-H12 & 119.4 & C32-C33-H36 & 109.2 \\
\hline C17-C20 & c 1.5341 & 1.526 & C10-C8-H12 & 120.0 & C32-C33-H37 & 112.1 \\
\hline C20-H21 & - & 1.111 & C6-C10-C8 & 119.3 & C35-C33-H36 & 110.4 \\
\hline C20-H22 & - & 1.098 & C6-C10-H13 & 120.3 & C35-C33-H37 & 113.5 \\
\hline C20-N23 & c 1.4625 & 1.467 & C8-C10-H13 & 120.4 & H36-C33-H37 & 107.4 \\
\hline $\mathrm{N} 23-\mathrm{C} 24$ & c 1.4629 & 1.465 & C1-C14-O15 & 125.8 & C32-C34-C38 & 104.6 \\
\hline N23-C28 & c 1.4623 & 1.465 & C1-C14-O16 & 110.7 & C32-C34-H39 & 110.7 \\
\hline C24-H25 & - & 1.097 & O15-C14-O16 & 123.4 & C32-C34-H40 & 109.4 \\
\hline C24-H26 & - & 1.109 & C14-O16-C17 & 117.5 & C38-C34-H39 & 113.3 \\
\hline C24-H27 & - & 1.095 & O16-C17-H18 & 106.2 & C38-C34-H40 & 110.6 \\
\hline C28-H29 & - & 1.097 & O16-C17-H19 & 109.5 & H39-C34-H40 & 108.2 \\
\hline C28-H30 & - & 1.097 & O16-C17-C20 & 109.3 & C33-C35-C38 & 105.9 \\
\hline C28-H31 & - & 1.109 & H18-C17-H19 & 109.1 & C33-C35-H41 & 110.4 \\
\hline C32-C33 & \multirow{5}{*}{$\begin{array}{l}\text { a } 1.522-1.539 \\
\text { b } 1.510-1.533\end{array}$} & 1.540 & H18-C17-C20 & 109.3 & C33-C35-H42 & 111.2 \\
\hline C32-C34 & & 1.537 & H19-C17-C20 & 113.2 & C38-C35-H41 & 110.2 \\
\hline C33-C35 & & 1.546 & $\mathrm{C} 17-\mathrm{C} 20-\mathrm{H} 21$ & 107.8 & C38-C35-H42 & 112.1 \\
\hline C34-C38 & & 1.546 & C17-C20-H22 & 108.1 & $\mathrm{H} 41-\mathrm{C} 35-\mathrm{H} 42$ & 107.0 \\
\hline C35-C38 & & 1.559 & C17-C20-N23 & 114.3 & C34-C38-C35 & 105.7 \\
\hline $\mathrm{C} 32-\mathrm{O} 45$ & $\begin{array}{l}\text { a } 1.444 \\
\text { b } 1.428\end{array}$ & 1.433 & $\mathrm{H} 21-\mathrm{C} 20-\mathrm{H} 22$ & 107.3 & C34-C38-H43 & 110.4 \\
\hline C33-H36 & - & 1.097 & H21-C20-N23 & 111.5 & C34-C38-H44 & 111.2 \\
\hline C33-H37 & - & 1.096 & H22-C20-N23 & 107.6 & C35-C38-H43 & 110.4 \\
\hline C34-H39 & - & 1.097 & C20-N23-C24 & 112.6 & C35-C38-H44 & 112.0 \\
\hline C34-H40 & - & 1.097 & C20-N23-C28 & 110.5 & H43-C38-H44 & 107.2 \\
\hline C35-H41 & - & 1.097 & C24-N23-C28 & 110.7 & C32-O45-H46 & 111.5 \\
\hline C35-H42 & - & 1.098 & C4-C3-C1-C32 & -100.6 & O45-H46”N23 & 164.1 \\
\hline C38-H43 & - & 1.096 & C5-C3-C1-C14 & -46.2 & O15-C14-C1-C32 & -74.9 \\
\hline C38-H44 & - & 1.098 & C14-C16-C17-C20 & 102.3 & O16-C14-C1-C32 & 102.2 \\
\hline $\mathrm{O} 45-\mathrm{H} 46$ & - & 0.980 & C1-C14-O16-C17 & -164.2 & $\mathrm{C} 14-\mathrm{C} 1-\mathrm{C} 32-\mathrm{O} 45$ & -59.1 \\
\hline \multirow[t]{5}{*}{$\mathrm{H} 466^{\cdots} \mathrm{N} 23$} & - & 2.027 & C14-C1-C32-C33 & 66.4 & C1-C32-O45-H46 & 66.4 \\
\hline & & & C17-C20-N23-C24 & -70.1 & C16-C17-C20-N23 & -62.4 \\
\hline & & & C17-C20-N23-C28 & 165.5 & C32-C34-C38-C35 & 24.0 \\
\hline & & & C5-C3-C4-C6 & -0.8 & C33-C35-C38-C34 & 0.6 \\
\hline & & & C3-C1-C32-C33 & -61.9 & O15-C14-O16-C17 & 12.9 \\
\hline
\end{tabular}

a, b, c, d and e Taken from Refs. Hirschler, 1994; Angelova, 1992; Sroczyński, 2017; Trotter, 1960; Palafox, 2000, respectively. 
In the literature, two molecular forms as $R$ and $S$ - are available for cyclopentolate. These structures are also known as enantiomers or optical isomers. Szwed et al. (2013) used experimental and computational (optimized molecular geometries) methods to determine the absolute configurations of enantiomers of cyclopentolate molecule. They reported optimized molecular structures for the three lowest-energy conformers with the B3LYP/aug-ccpVDZ level of $(R)$ cyclopentolate (Szwed et al, 2013). In this study, considering the molecular geometric forms obtained by Szwed et al. (2013), we determined the four conformational forms for the cylopentolate molecule. In our study, as shown in Figure 1, the calculated energy values for four lowest-energy conformational forms of cyclopentolate molecule were obtained as -943.40481341 Hartrees (for conformer I), -

943.40439951 Hartrees (for conformer II), 943.40431503 Hartrees (for conformer III) and -943.40317790 Hartrees (for conformer IV) with the B3LYP/aug-cc-pVDZ level, respectively. As indicated by these computed energy values, conformer I is the most stable molecular form. Therefore, in this study, detailed structural and spectroscopic analyses were performed for conformer I.

The C-C bond lengths in phenyl ring of the title molecule were calculated at the interval of 1.397-1.405 $\AA$, while they recorded between 1.36-1.41 $\AA$ for benzene derivatives in the literature (Trotter, 1960). Additionally, the $\mathrm{C}-\mathrm{C}$ and $\mathrm{C}-\mathrm{H}$ bond lengths obtained with some quantum chemical computational levels of the benzene ring were calculated at the intervals of 1.3920-1.3990 $\AA$ (1.3920-1.3990 $\AA$ (exp.)) and 1.0831-1.1010 $\AA$ (1.0831$1.1010 \AA$ (exp.)) by Palafox (2000) respectively. Similarly, the C32-C33, C32C34, C33-C35, C34-C38, C35-C38 and C32-
O45 bond lengths in 1-hydroxycyclopentyl group were computed as $1.540 \AA, 1.537 \AA$, $1.546 \AA, 1.546 \AA, 1.559 \AA$ and $1.433 \AA$, respectively.

Hirschler et al. (1994) reported values at the interval of 1.522-1.539 $\AA$ for $\mathrm{C}-\mathrm{C}$ bond lengths and $1.444 \AA$ for $\mathrm{C}-\mathrm{O}$ bond length in 1hydroxycyclopentyl group in $(1 \mathrm{R}, 3 \mathrm{R})$ 2[(2R,4R,5R)-3-(p-chlorophenylsulfonyl)-4methyl-5-phenyl-1,3-oxazolidin-2-yl]-5-\{2(1R,2S)-[(2R,4R,5R)-3-(pchlorophenylsulfonyl)-4-methyl-5-phenyl1,3-oxazo-lidin-2-yl]-1hydroxycyclopentyl \}-1-cyclopentanone (C42H44Cl2N2O8S2) molecule. Likewise, the $\mathrm{C}-\mathrm{C}$ and $\mathrm{C}-\mathrm{O}$ bond lengths in 1hydroxycyclopentyl group of $( \pm)$ diethyl ester of (1-hydroxycyclopentyl) (2methylphenyl)methylphosphonic acid were measured between 1.510-1.533 $\AA$ and at 1.428 $\AA$ by Angelova et al. (1992) The C1-C3, C1C14 and C1-C32 bond lengths were theoretically obtained at $1.523 \AA, 1.521 \AA$, and $1.580 \AA$, respectively. The $\mathrm{C}-\mathrm{O}$ bond lengths were calculated as $1.215 \AA$ for $\mathrm{C} 14=\mathrm{O} 15,1.357 \AA$ for $\mathrm{C} 14-\mathrm{O} 16$ and $1.443 \AA$ for C17-O16. The C24,28-N23, C20-N23 and $\mathrm{C} 17-\mathrm{C} 20$ bond lengths in 2(dimethylamino)ethyl group in the title molecule were computed at $1.465 \AA, 1.467 \AA$ and $1.526 \AA$. Sroczynski and Malinowski (2017) computed the $\mathrm{N}-\mathrm{CH}_{3}$ bond lengths as $1.4629 \AA$ and $1.4623 \AA$, the N-C bond length as $1.4625 \AA$ and the $\mathrm{CH}_{2}-\mathrm{CH}_{2}$ bond length as $1.5341 \AA$ in 2-(dimethylamino)ethyl group of 2-[2-(dimethylamino)ethyl]-6-methoxy-4-

(pyridin-2-yl)-1(2H)-phthalazinone molecule with the B3LYP/6-311++G(d,p) level (Sroczyński and Malinowski, 2017). The C-H bond lengths in the title molecule were calculated at the interval of 1.087-1.111 $\AA$. Likewise, the O45-H46 bond length was computed as $0.980 \AA$. 


\begin{tabular}{|c|c|c|c|c|c|c|c|}
\hline \multicolumn{2}{|c|}{ Exp. freq. $\left(\mathrm{cm}^{-1}\right)$} & \multirow{2}{*}{ Assignment (PED\%) } & \multicolumn{5}{|c|}{ The computed parameters } \\
\hline IR & Raman & & Mode & Freq. & $\mathrm{IIR}_{\mathrm{IR}}$ & $S_{\text {Raman }}$ & $\begin{array}{l}{ }^{\mathrm{a}} \mathrm{I}_{\text {Raman }} \\
\left(\times 10^{-36}\right)\end{array}$ \\
\hline 3230 & - & vOH(100) & $v_{132}$ & 3386 & 723.72 & 297.60 & 36.41 \\
\hline 3105 & - & $v \mathrm{CH}(100)$ in phenyl & $v_{131}$ & 3123 & 1.69 & 75.08 & 11.18 \\
\hline 3090 & - & $v \mathrm{CH}(91)$ in phenyl & $v_{130}$ & 3099 & 21.50 & 294.58 & 44.67 \\
\hline- & - & $v \mathrm{CH}(87)$ in phenyl & $v_{129}$ & 3087 & 24.16 & 78.91 & 12.08 \\
\hline- & - & $v \mathrm{CH}(94)$ in phenyl & $v_{128}$ & 3076 & 1.70 & 106.16 & 16.38 \\
\hline- & 3068 & $v \mathrm{CH}(95)$ in phenyl & $v_{127}$ & 3069 & 4.45 & 27.06 & 4.20 \\
\hline- & 3051 & $v_{\text {as }} \mathrm{CH}_{2}(82)$ & $v_{126}$ & 3052 & 6.12 & 44.89 & 7.05 \\
\hline 3039 & 3045 & $v_{\mathrm{as}} \mathrm{CH}_{3}(81)$ & $v_{125}$ & 3041 & 10.53 & 20.54 & 3.25 \\
\hline- & - & $v_{\mathrm{as}} \mathrm{CH}_{3}(97)$ & $v_{124}$ & 3029 & 15.49 & 37.24 & 5.95 \\
\hline- & - & $v_{\mathrm{as}} \mathrm{CH}_{2}(96)$ in cycl. & $v_{123}$ & 3028 & 75.87 & 44.12 & 7.06 \\
\hline 3017 & 3020 & $v_{\text {as }} \mathrm{CH}_{2}(97)$ in cycl. & $v_{122}$ & 3022 & 13.03 & 52.42 & 8.43 \\
\hline- & - & $v_{\text {as }} \mathrm{CH}_{2}(86)$ in cycl. & $v_{121}$ & 3009 & 4.09 & 120.98 & 19.63 \\
\hline- & - & vCH(98) & $v_{120}$ & 2998 & 5.01 & 25.49 & 4.17 \\
\hline- & - & $v_{\mathrm{as}} \mathrm{CH}_{3}(55)+v_{\mathrm{s}} \mathrm{CH}_{2}(24)$ & $v_{119}$ & 2994 & 0.44 & 213.06 & 34.98 \\
\hline- & - & $v_{\text {as }} \mathrm{CH}_{2}(82)$ in cycl. & $v_{118}$ & 2993 & 4.51 & 30.09 & 4.95 \\
\hline 2990 & 2990 & $v_{\mathrm{s}} \mathrm{CH}_{2}(68)+v_{\mathrm{as}} \mathrm{CH}_{3}(17)$ & $v_{117}$ & 2992 & 50.38 & 46.02 & 7.57 \\
\hline- & - & $v_{\mathrm{s}} \mathrm{CH}_{2}(72)+v_{\mathrm{as}} \mathrm{CH}_{3}(21)$ & $v_{116}$ & 2988 & 22.68 & 67.99 & 11.21 \\
\hline- & - & $v_{\text {as }} \mathrm{CH}_{3}(82)$ & $v_{115}$ & 2986 & 13.18 & 28.76 & 4.75 \\
\hline- & - & $v_{\mathrm{s}} \mathrm{CH}_{2}(95)$ in cycl. & $v_{114}$ & 2980 & 24.42 & 399.70 & 66.36 \\
\hline 2964 & 2962 & $v_{\mathrm{s}} \mathrm{CH}_{2}(81)$ in cycl. & $v_{113}$ & 2972 & 39.52 & 13.57 & 2.27 \\
\hline 2953 & 2943 & $v_{\mathrm{s}} \mathrm{CH}_{2}(98)$ in cycl. & $v_{112}$ & 2969 & 61.32 & 32.25 & 5.40 \\
\hline 2927 & 2915 & $v_{\mathrm{s}} \mathrm{CH}_{2}(96)$ in cycl. & $v_{111}$ & 2962 & 14.83 & 50.40 & 8.48 \\
\hline 2885 & - & $v_{\mathrm{s}} \mathrm{CH}_{3}(87)$ & $v_{110}$ & 2862 & 229.75 & 424.61 & 77.16 \\
\hline 2880 & 2872 & $v_{\mathrm{s}} \mathrm{CH}_{3}(92)$ & $v_{109}$ & 2854 & 35.41 & 46.67 & 8.53 \\
\hline- & - & $v_{\mathrm{s}} \mathrm{CH}_{2}(91)$ & $v_{108}$ & 2837 & 66.88 & 121.78 & 22.55 \\
\hline 1735 & 1734 & $v \mathrm{C}=\mathrm{O}(87)$ & $v_{107}$ & 1710 & 138.02 & 5.29 & 2.54 \\
\hline 1605 & 1604 & {$[v \mathrm{CC}(52)+\delta \mathrm{HCC}(16)]$ in phenyl } & $v_{106}$ & 1595 & 7.87 & 50.21 & 27.01 \\
\hline 1584 & 1584 & {$[v \mathrm{CC}(56)+\delta \mathrm{CCC}(23)]$ in phenyl } & $v_{105}$ & 1573 & 0.98 & 10.00 & 5.51 \\
\hline 1472 & 1474 & {$[\delta \mathrm{HCC}(61)+\delta \mathrm{CCC}(11)]$ in phenyl } & $v_{104}$ & 1470 & 15.45 & 0.42 & 0.25 \\
\hline- & - & $\delta_{\mathrm{s}} \mathrm{CH}_{2}(77)$ in cycl. & $v_{103}$ & 1463 & 3.79 & 1.82 & 1.12 \\
\hline 1456 & 1454 & $\delta_{\mathrm{s}} \mathrm{CH}_{3}(41)+\delta_{\mathrm{s}} \mathrm{CH}_{2}(24)$ & $v_{102}$ & 1450 & 14.09 & 1.30 & 0.81 \\
\hline 1452 & 1452 & $\delta_{\mathrm{s}} \mathrm{CH}_{3}(49)+\delta_{\mathrm{s}} \mathrm{CH}_{2}(12)$ & $v_{101}$ & 1446 & 10.29 & 11.54 & 7.24 \\
\hline- & - & $\delta_{\mathrm{s}} \mathrm{CH}_{3}(24)+\delta_{\mathrm{s}} \mathrm{CH}_{2}(21)$ in cycl. and aliphatic & $v_{100}$ & 1440 & 4.77 & 3.54 & 2.23 \\
\hline- & - & $\delta_{\mathrm{s}} \mathrm{CH}_{2}(43)$ in cycl. $+\delta_{\mathrm{s}} \mathrm{CH}_{2}(10)$ & $v_{99}$ & 1438 & 6.03 & 1.32 & 0.83 \\
\hline- & - & $\delta_{\mathrm{s}} \mathrm{CH}_{2}(54)$ in cycl. & v98 & 1438 & 3.36 & 3.50 & 2.21 \\
\hline- & - & $\delta_{\mathrm{s}} \mathrm{CH}_{3}(31)+\delta_{\mathrm{s}} \mathrm{CH}_{2}(30)$ & $v_{97}$ & 1435 & 31.07 & 5.26 & 3.34 \\
\hline- & - & {$[\delta \mathrm{HCC}(35)+v \mathrm{CC}(10)]$ in phenyl } & $v_{96}$ & 1429 & 9.37 & 2.59 & 1.66 \\
\hline- & - & $\delta_{\mathrm{s}} \mathrm{CH}_{2}(47)$ in cycl. & $v_{95}$ & 1426 & 2.65 & 9.40 & 6.03 \\
\hline
\end{tabular}




\begin{tabular}{|c|c|c|c|c|c|c|c|}
\hline- & 1421 & $\delta_{\mathrm{s}} \mathrm{CH}_{3}(75)$ & $v_{94}$ & 1425 & 2.31 & 6.25 & 4.01 \\
\hline 1417 & 1416 & $\delta_{\mathrm{s}} \mathrm{CH}_{3}(22)+\delta_{\mathrm{s}} \mathrm{CH}_{2}(20)$ & $v_{93}$ & 1422 & 3.22 & 4.34 & 2.79 \\
\hline 1399 & - & $\delta_{\mathrm{s}} \mathrm{CH}_{3}(38)$ (sym. bending) $+\delta_{\mathrm{s}} \mathrm{CH}_{2}(13)$ & $v_{92}$ & 1406 & 1.47 & 4.58 & 3.00 \\
\hline- & - & $\delta \mathrm{HOC}(37)+\delta_{\mathrm{s}} \mathrm{CH}_{3}(27)$ (sym. bending) & $v_{91}$ & 1386 & 41.04 & 1.07 & 0.71 \\
\hline 1375 & 1372 & $\delta \mathrm{HOC}(34)+\delta_{\mathrm{s}} \mathrm{CH}_{3}(11)$ (sym. bending) & $v_{90}$ & 1379 & 25.58 & 2.71 & 1.83 \\
\hline- & 1361 & $\mathrm{wCH}_{2}(25)+\delta \mathrm{H}_{2} \mathrm{C}_{1} \mathrm{C}_{3}(13)$ & $v_{89}$ & 1362 & 10.10 & 0.59 & 0.40 \\
\hline 1354 & - & $\mathrm{wCH}_{2}(19)$ & $v_{88}$ & 1350 & 71.97 & 0.99 & 0.69 \\
\hline 1322 & - & $\delta \mathrm{H}_{2} \mathrm{C}_{1} \mathrm{C}_{3}(15)+\mathrm{wCH} \mathrm{H}_{2}(15)+\delta \mathrm{HCC}(10)$ in phenyl & $2 v_{87}$ & 1328 & 12.25 & 0.63 & 0.45 \\
\hline- & - & $\mathrm{wCH}_{2}(34)$ in cycl. & $v_{86}$ & 1316 & 4.89 & 0.40 & 0.29 \\
\hline 1307 & 1299 & {$[\delta \mathrm{HCC}(42)+v C C(40)]$ in phenyl } & $v_{85}$ & 1311 & 2.12 & 1.01 & 0.73 \\
\hline 1289 & - & $\mathrm{wCH}_{2}(29)$ in cycl. & $v_{84}$ & 1289 & 6.90 & 1.15 & 0.86 \\
\hline- & - & $\mathrm{wCH}_{2}(21)$ in cycl. & $v_{83}$ & 1284 & 31.42 & 2.67 & 2.00 \\
\hline- & - & $\mathrm{wCH}_{2}(15)$ in cycl. $+\delta \mathrm{HCC}(15)$ in phenyl & $v_{82}$ & 1282 & 60.88 & 0.39 & 0.29 \\
\hline- & - & $\mathrm{tCH}_{2}(21)$ & $v_{81}$ & 1268 & 12.93 & 5.05 & 3.86 \\
\hline 1265 & 1264 & $\mathrm{wCH}_{2}(32)$ in cycl. & $v_{80}$ & 1267 & 3.65 & 1.48 & 1.13 \\
\hline- & - & $\mathrm{tCH}_{2}(30)+v \mathrm{CN}(14)+\rho \mathrm{CH}_{3}(11)$ & v79 & 1260 & 17.23 & 2.86 & 2.20 \\
\hline 1254 & 1258 & $\mathrm{wCH}_{2}(15)$ in cycl. & V78 & 1250 & 1.30 & 5.49 & 4.29 \\
\hline 1236 & 1234 & $\mathrm{tCH}_{2}(22)$ & v77 & 1239 & 8.61 & 3.61 & 2.85 \\
\hline- & - & $\mathrm{tCH}_{2}(67)$ in cycl. & $v_{76}$ & 1215 & 2.38 & 2.44 & 1.98 \\
\hline 1199 & - & $\mathrm{tCH}_{2}(14)$ in cycl. & v75 & 1197 & 63.70 & 13.84 & 11.51 \\
\hline 1186 & 1186 & $v \mathrm{CN}(15)$ & $v_{74}$ & 1188 & 12.37 & 9.16 & 7.70 \\
\hline- & - & - & $v_{73}$ & 1180 & 34.31 & 4.08 & 3.46 \\
\hline 1177 & - & $\mathrm{tCH}_{2}(22)$ in cycl. & $v_{72}$ & 1177 & 4.00 & 19.24 & 16.38 \\
\hline- & 1162 & $\mathrm{tCH}_{2}(23)$ in cycl. $+v \mathrm{C}_{1} \mathrm{C}_{3}(10)$ & $v_{71}$ & 1168 & 2.83 & 21.48 & 18.49 \\
\hline- & 1158 & {$[\delta \mathrm{HCC}(78)+v \mathrm{CC}(17)]$ in phenyl } & $v_{70}$ & 1164 & 1.74 & 4.95 & 4.28 \\
\hline 1149 & - & $\rho \mathrm{CH}_{3}(48)$ & $v_{69}$ & 1143 & 27.54 & 11.69 & 10.39 \\
\hline- & - & {$[\delta \mathrm{HCC}(76)+v \mathrm{CC}(14)]$ in phenyl } & $v_{68}$ & 1136 & 0.05 & 3.93 & 3.52 \\
\hline- & - & $\nu \mathrm{C}_{14} \mathrm{O}_{16}(33)+\delta \mathrm{OCO}(12)$ & $v_{67}$ & 1124 & 312.86 & 3.05 & 2.77 \\
\hline 1101 & 1090 & $v \mathrm{CC}(22)$ in cycl. & $v_{66}$ & 1089 & 32.37 & 8.35 & 7.94 \\
\hline 1080 & - & $\rho \mathrm{CH}_{3}(66)$ & $v_{65}$ & 1078 & 4.79 & 2.12 & 2.05 \\
\hline- & - & {$[\delta \mathrm{HCC}(40)+v \mathrm{CC}(36)]$ in phenyl } & $v_{64}$ & 1067 & 10.50 & 1.37 & 1.34 \\
\hline 1061 & 1065 & $v \mathrm{CC}(18)$ in cycl. & $v_{63}$ & 1059 & 15.09 & 8.17 & 8.08 \\
\hline 1049 & 1052 & $\rho \mathrm{CH}_{2}(17)+v \mathrm{C}_{17} \mathrm{C}_{20}(10)$ & $v_{62}$ & 1053 & 2.14 & 5.01 & 4.99 \\
\hline 1034 & 1038 & $v \mathrm{CN}(29)+\rho \mathrm{CH}_{3}(18)$ & $v_{61}$ & 1044 & 15.89 & 2.32 & 2.34 \\
\hline 1028 & 1031 & {$[v C C(31)+\delta H C C(14)]$ in phenyl } & $v_{60}$ & 1022 & 1.06 & 19.12 & 19.89 \\
\hline- & - & $v \mathrm{CN}(43)+\rho \mathrm{CH}_{3}(10)$ & $v_{59}$ & 1020 & 17.76 & 3.23 & 3.36 \\
\hline 1010 & 1007 & $v \mathrm{CC}(28)$ in cycl. & $v_{58}$ & 1014 & 10.92 & 9.26 & 9.73 \\
\hline- & - & {$\left[\tau \operatorname{CCCC}(26)+\delta \mathrm{CCC}(14)+\rho \mathrm{CH}_{2}(11)\right]$ in cycl. } & $v_{57}$ & 1004 & 2.10 & 2.04 & 2.17 \\
\hline 991 & 990 & $v \mathrm{C}_{17} \mathrm{O}_{16}(21)+v \mathrm{CC}(15)$ in cycl. & $v_{56}$ & 997 & 36.64 & 7.59 & 8.17 \\
\hline- & - & $\mathrm{tCH}_{2}(19)+v \mathrm{C}_{32} \mathrm{O}_{45}(13)$ & $v_{55}$ & 986 & 14.98 & 15.76 & 17.23 \\
\hline- & 973 & Sphenyl(85) & $v_{54}$ & 980 & 1.69 & 34.95 & 38.53 \\
\hline 967 & - & {$[\tau \operatorname{HCCC}(57)+\tau \mathrm{CCCC}(22)]$ in phenyl } & $v_{53}$ & 967 & 0.35 & 1.76 & 1.98 \\
\hline 952 & 953 & $\tau \mathrm{HCCC}(79)$ in phenyl & $v_{52}$ & 952 & 0.31 & 0.53 & 0.60 \\
\hline
\end{tabular}




\begin{tabular}{|c|c|c|c|c|c|c|c|}
\hline- & 929 & $v C C(40)$ in cycl. & $v_{51}$ & 947 & 7.39 & 0.80 & 0.92 \\
\hline 921 & 920 & $v \mathrm{CN}(35)+\rho \mathrm{CH}_{2}(12)$ & $v_{50}$ & 934 & 26.16 & 2.85 & 3.35 \\
\hline- & 912 & $\tau \mathrm{HCCC}(11)$ in phenyl & $v_{49}$ & 913 & 28.48 & 1.93 & 2.35 \\
\hline- & - & $v \mathrm{C}_{32} \mathrm{O}_{45}(11)+v \mathrm{C}_{1} \mathrm{C}_{32}(11)$ & $v_{48}$ & 900 & 11.42 & 1.94 & 2.40 \\
\hline 893 & 890 & $\tau \mathrm{HCCC}(55)$ in phenyl & $v_{47}$ & 897 & 12.92 & 3.01 & 3.75 \\
\hline 861 & 870 & Scycl.(38) & $v_{46}$ & 865 & 2.06 & 14.76 & 19.28 \\
\hline- & 867 & $v \mathrm{C}_{17} \mathrm{O}_{16}(22)+\delta \mathrm{O}_{16} \mathrm{C}_{24} \mathrm{C}_{1}(11)$ & $v_{45}$ & 857 & 1.18 & 12.21 & 16.16 \\
\hline- & 838 & $v \mathrm{C}_{17} \mathrm{O}_{16}(22)+\gamma \mathrm{OCOC}(12)$ & $v_{44}$ & 846 & 2.23 & 1.71 & 2.30 \\
\hline- & 829 & $\tau \mathrm{HCCC}(98)$ in phenyl & $v_{43}$ & 827 & 0.22 & 0.58 & 0.80 \\
\hline- & - & {$\left[v \mathrm{CC}(24)+\rho \mathrm{CH}_{2}(10)\right]$ in cycl. } & $v_{42}$ & 799 & 3.01 & 9.09 & 13.23 \\
\hline 786 & 790 & Scycl.(10) & $v_{41}$ & 791 & 1.39 & 1.21 & 1.78 \\
\hline- & - & $v \mathrm{NC}(16)$ & $v_{40}$ & 774 & 14.11 & 0.77 & 1.17 \\
\hline 750 & 753 & $v \mathrm{NC}(41)+v \mathrm{C}_{17} \mathrm{C}_{20}(11)$ & $v_{39}$ & 763 & 9.18 & 6.77 & 10.49 \\
\hline 736 & 742 & $\tau \mathrm{HCCC}(13)$ in phenyl $+\gamma \mathrm{OCOC}(12)+\delta \mathrm{OCO}(10)$ & $v_{38}$ & 734 & 6.61 & 0.78 & 1.27 \\
\hline- & 720 & $\delta \mathrm{OCO}(17)+\tau \mathrm{HCCC}(10)$ in phenyl & $v_{37}$ & 719 & 39.70 & 2.00 & 3.36 \\
\hline 703 & - & {$\left[\rho \mathrm{CH}_{2}(25)+v \mathrm{CC}(13)\right]$ in cycl. } & $v_{36}$ & 706 & 2.90 & 4.53 & 7.80 \\
\hline- & - & {$[\tau \operatorname{HCCC}(54)+\tau \mathrm{CCCC}(29)]$ in phenyl } & $v_{35}$ & 690 & 39.03 & 0.23 & 0.40 \\
\hline- & - & $\tau \mathrm{HOCC}(72)$ & $v_{34}$ & 682 & 34.96 & 0.32 & 0.58 \\
\hline 643 & 644 & 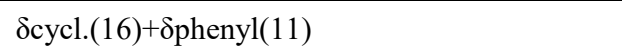 & $v_{33}$ & 641 & 13.96 & 0.97 & 1.91 \\
\hline- & 640 & Scycl.(29) & $v_{32}$ & 638 & 0.12 & 0.63 & 1.24 \\
\hline- & 621 & 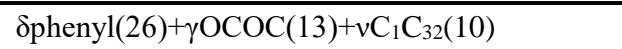 & $v_{31}$ & 611 & 1.06 & 3.37 & 7.06 \\
\hline 589 & 592 & Sphenyl(85) & $v_{30}$ & 577 & 19.88 & 3.85 & 8.71 \\
\hline 552 & 554 & $\delta \mathrm{NC}_{20} \mathrm{C}_{17}(23)+\delta \mathrm{C}_{20} \mathrm{C}_{18} \mathrm{O}_{16}(20)$ & $v_{29}$ & 536 & 1.54 & 0.33 & 0.83 \\
\hline 501 & 502 & 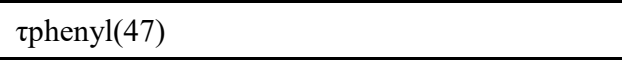 & $v_{28}$ & 492 & 5.05 & 0.72 & 2.02 \\
\hline 451 & 459 & $\delta \mathrm{O}_{45} \mathrm{C}_{32} \mathrm{C}_{34}(31)+\gamma \mathrm{O}_{45} \mathrm{C}_{1} \mathrm{C}_{32} \mathrm{C}_{34}(23)$ & $v_{27}$ & 443 & 2.72 & 1.03 & 3.38 \\
\hline 422 & 424 & $\delta \mathrm{C}_{24} \mathrm{NC}_{28}(53)$ & $v_{26}$ & 425 & 2.29 & 0.36 & 1.23 \\
\hline- & 410 & {$[\tau \operatorname{CCCC}(75)+\tau \operatorname{HCCC}(20)]$ in phenyl } & $v_{25}$ & 407 & 0.33 & 0.03 & 0.10 \\
\hline 403 & - & $\gamma \mathrm{C}_{34} \mathrm{C}_{1} \mathrm{C}_{33} \mathrm{C}_{32}(19)$ & $v_{24}$ & 401 & 1.04 & 1.53 & 5.78 \\
\hline- & 392 & $\delta \mathrm{C}_{20} \mathrm{NC}_{24}(33)+\rho \mathrm{CH}_{2}(11)$ & $v_{23}$ & 397 & 20.10 & 0.75 & 2.88 \\
\hline- & - & $\delta \mathrm{COC}(26)+\gamma \mathrm{C}_{28} \mathrm{C}_{20} \mathrm{C}_{44} \mathrm{~N}(18)+\delta \mathrm{OCO}(10)$ & $v_{22}$ & 386 & 13.45 & 0.94 & 3.73 \\
\hline- & - & $\delta \mathrm{C}_{20} \mathrm{C}_{17} \mathrm{O}_{16}(11)+\gamma \mathrm{C}_{28} \mathrm{C}_{20} \mathrm{C}_{44} \mathrm{~N}(21)$ & $v_{21}$ & 345 & 1.00 & 1.50 & 7.06 \\
\hline- & 321 & $\gamma \mathrm{O}_{45} \mathrm{C}_{1} \mathrm{C}_{34} \mathrm{C}_{33}(30)+\delta \mathrm{O}_{45} \mathrm{C}_{32} \mathrm{C}_{34}(11)$ & $v_{20}$ & 319 & 8.24 & 0.63 & 3.32 \\
\hline- & - & $\delta \mathrm{O}_{16} \mathrm{C}_{14} \mathrm{C}_{1}(18)+\delta \mathrm{C}_{1} \mathrm{C}_{3} \mathrm{C}_{5}(18)$ & $v_{19}$ & 303 & 1.07 & 1.19 & 6.83 \\
\hline- & 283 & $v \mathrm{C}_{1} \mathrm{C}_{3}(22)+\tau \mathrm{CH}_{3}(14)+\delta \mathrm{O}_{16} \mathrm{C}_{14} \mathrm{C}_{1}(12)$ & $v_{18}$ & 275 & 0.81 & 3.85 & 25.57 \\
\hline- & - & $\tau \mathrm{CH}_{3}(26)$ & $v_{17}$ & 256 & 1.27 & 1.22 & 9.02 \\
\hline- & 249 & $\tau \mathrm{CH}_{3}(15)+\delta \mathrm{C}_{1} \mathrm{C}_{32} \mathrm{C}_{33}(13)$ & $v_{16}$ & 248 & 0.45 & 1.33 & 10.44 \\
\hline- & - & $\tau$ phenyl(14) $+\tau \mathrm{CH}_{3}(11)$ & $v_{15}$ & 241 & 1.21 & 1.41 & 11.55 \\
\hline- & - & $\tau \mathrm{CH}_{3}(19)$ & $v_{14}$ & 238 & 1.35 & 1.01 & 8.43 \\
\hline- & - & $\delta \mathrm{NCC}(28)$ & $v_{13}$ & 211 & 1.52 & 0.28 & 2.87 \\
\hline- & - & $\delta \mathrm{C}_{1} \mathrm{C}_{3} \mathrm{C}_{5}(32)+\tau$ cycl.(13) & $v_{12}$ & 182 & 2.55 & 0.46 & 5.98 \\
\hline- & 146 & $\tau$ cycl.(36) $+\tau \mathrm{C}_{17} \mathrm{O}_{26} \mathrm{C}_{14} \mathrm{C}_{1}(18)$ & $v_{11}$ & 152 & 1.92 & 0.48 & 8.36 \\
\hline- & - & $\gamma \mathrm{C}_{14} \mathrm{C}_{32} \mathrm{O}_{1} \mathrm{C}_{3}(13)+\tau \mathrm{CNCC}(11)$ & $v_{10}$ & 129 & 0.77 & 1.29 & 30.43 \\
\hline- & 112 & $\tau \mathrm{CNCC}(31)+\gamma \mathrm{C}_{14} \mathrm{C}_{32} \mathrm{O}_{1} \mathrm{C}_{3}(11)$ & $v_{9}$ & 120 & 0.55 & 0.08 & 2.08 \\
\hline- & - & $\tau \mathrm{C}_{20} \mathrm{C}_{17} \mathrm{O}_{16} \mathrm{C}_{14}(44)$ & $v_{8}$ & 100 & 0.38 & 0.32 & 11.91 \\
\hline
\end{tabular}




\begin{tabular}{|c|l|l|c|c|c|c|c|}
\hline- & - & $\tau \mathrm{NCCC}(13)+\tau \mathrm{C}_{20} \mathrm{C}_{17} \mathrm{O}_{16} \mathrm{C}_{14}(13)+\delta \mathrm{C}_{3} \mathrm{C}_{1} \mathrm{C}_{32}(11)$ & $v_{7}$ & 86 & 0.66 & 1.92 & 93.62 \\
\hline- & - & $\tau \mathrm{C}_{17} \mathrm{O}_{16} \mathrm{C}_{14} \mathrm{C}_{1}(26)+\tau \mathrm{C}_{33} \mathrm{C}_{32} \mathrm{C}_{1} \mathrm{C}_{3}(21)$ & $v_{6}$ & 79 & 0.42 & 0.07 & 4.04 \\
\hline- & - & $\tau \mathrm{C}_{32} \mathrm{C}_{1} \mathrm{C}_{3} \mathrm{C}_{4}(22)+\tau c y c l .(15)$ & $v_{5}$ & 71 & 0.46 & 2.47 & 172.03 \\
\hline- & - & $\tau c y c l .(19)+\delta \mathrm{C}_{3} \mathrm{C}_{1} \mathrm{C}_{32}(13)+\rho \mathrm{CH}_{2}(12)$ in cycl. & $v_{4}$ & 70 & 0.21 & 1.35 & 96.47 \\
\hline- & - & $\tau \mathrm{C}_{32} \mathrm{C}_{1} \mathrm{C}_{3} \mathrm{C}_{4}(56)$ & $v_{3}$ & 50 & 0.03 & 3.54 & 464.10 \\
\hline- & - & $\tau \mathrm{O}_{16} \mathrm{C}_{14} \mathrm{C}_{1} \mathrm{C}_{32}(24)+\tau \mathrm{C}_{33} \mathrm{C}_{32} \mathrm{C}_{1} \mathrm{C}_{3}(21)+\tau \mathrm{C}_{17} \mathrm{O}_{16} \mathrm{C}_{14} \mathrm{C}_{1}(1$ & $v_{2}$ & 42 & 1.99 & 0.99 & 180.94 \\
\hline- & - & $\tau)+\delta \mathrm{C}_{3} \mathrm{C}_{1} \mathrm{C}_{14}(12)$ & $v_{1}$ & 34 & 0.46 & 0.16 & 45.87 \\
\hline
\end{tabular}

$\mathbf{R}^{2}$ and RMSD values are $\mathbf{0 . 9 9 9 3 3}$ and $\mathbf{2 3 . 4} \mathbf{~ c m}^{-1}$ for IR wavenumbers and $\mathbf{0 . 9 9 9 8 4}$ and $\mathbf{1 0 . 6} \mathbf{~ c m}^{-1}$ for Raman wavenumbers, respectively.

$\mathrm{s}$, symmetric; as, asymmetric; $v$, stretching; $\delta$, in-plane bending; $\tau$, torsion; $\gamma$, out-of-plane bending; $\delta$, scissoring and symmetric bending; $\rho$, rocking; t, twisting; w, wagging; 1-hydroxycyclopentyl, cycl.; IIR, IR intensity (km/mol); SRaman, Raman scattering activity $\left(\AA^{4} / \mathrm{amu}\right) ; \mathrm{PED}$, potential energy distribution.

${ }^{a}$ Raman intensities (IRaman) and its spectrum were obtained by chemcraft program (www.chemcraftprog.com).

There is weak intramolecular hydrogen bond interaction in cyclopentolate molecule. This intramolecular hydrogen interaction is $\mathrm{O} 45$ H46...N23. The H46...N23 bond length and $\mathrm{O} 45-\mathrm{H} 46 \ldots \mathrm{N} 23$ bond angle in this weak interaction were theoretically obtained as $2.027 \AA$ and $164.1^{\circ}$.

The C3-C1-C14, C3-C1-C32 and C14-C1$\mathrm{C} 32$ bond angles were calculated as $113.0^{\circ}$, $116.2^{\circ}$ and $108.1^{\circ}$, respectively. The C-C-C bond angles in the phenyl ring of the title compound were calculated at the interval of $118.2^{\circ}-121.3^{\circ}$, while these bond angles in 1 hydroxycyclopentyl group were theoretically obtained at the interval of $102.6^{\circ}-105.9^{\circ}$. The C1-C32-O45, C33-C32-O45, C34-C32-O45, C1-C14-O15, C1-C14-O16, O15-C14-O16, C14-O16-C17 and O16-C17-C20 containing oxygen atoms were computed at $108.5^{\circ}$, $110.5^{\circ}, 105.4^{\circ}, 125.8^{\circ}, 110.7^{\circ}, 123.4^{\circ}, 117.5^{\circ}$ and $109.3^{\circ}$, while the C17-C20-N23, C20N23-C24, C20-N23-C28 and C24-N23-C28 bond angles in 2-(dimethylamino)ethyl group were theoretically found at $114.3^{\circ}, 112.6^{\circ}$, $110.5^{\circ}$ and $110.7^{\circ}$, respectively.
It can be inferred from the optimized molecular structure and computed dihedral angles for conformer I that cyclopentolate is non-planar. As a result, the C4-C3-C1-C32, C5-C3-C1-C14, C3-C1-C32-C33 and O16C14-C1-C32 dihedral angles were calculated as $-100.6^{\circ},-46.2^{\circ},-61.9^{\circ}$ and $102.2^{\circ}$, while the C14-O16-C17-C20, O16-C17-C20-N23, C17-C20-N23-C24 and C17-C20-N23-C28 dihedral angles were computed as $102.3^{\circ}$, $62.4^{\circ},-70.1^{\circ}$ and $165.5^{\circ}$, respectively.

\subsection{Vibrational Frequency Analyses}

In the following discussion, the cyclopentolate molecule is experimentally examined. The observed and calculated vibrational frequencies, calculated FT-IR intensities, Raman scattering activities and vibrational assignments of the title molecule are given in Table 2. The experimental and simulated IR spectra of cyclopentolate are shown in Figure 2 and the experimental and simulated Raman spectra of cyclopentolate are shown in Figure 3. The computations of harmonic wavenumbers, IR intensities and Raman activities were performed with the B3LYP/aug-cc-pVDZ level. Cyclopentolate 
consists of 46 atoms and accordingly it has 132 modes of vibrations. The linear correlation coefficient $\left(\mathrm{R}^{2}\right)$ and root-meansquare deviation (RMSD) values between the experimental and computed vibrational frequencies were found as 0.99933 and 23.4 $\mathrm{cm}^{-1}$ for IR wavenumbers and 0.99983 and $10.6 \mathrm{~cm}^{-1}$ for Raman ones, respectively. The experimental and simulated IR and Raman spectra of the title compound are given in Figures 2 and 3, respectively. In the present research, $\mathrm{C}-\mathrm{H}, \mathrm{C}-\mathrm{O}, \mathrm{C}-\mathrm{C}$ and $\mathrm{C}-\mathrm{N}$ vibrations were examined. As seen from Table 2, the experimental and calculated vibrational wavenumbers are in good agreement.

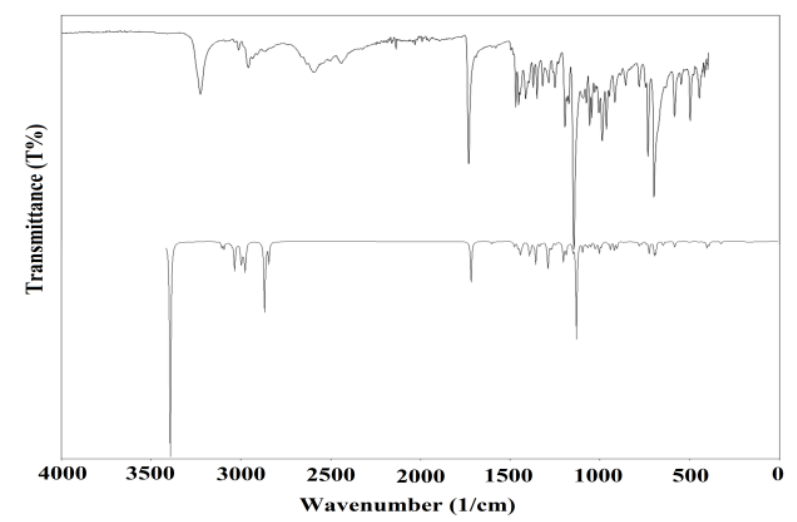

Figure 2. The experimental and simulated IR spectra of cyclopentolate

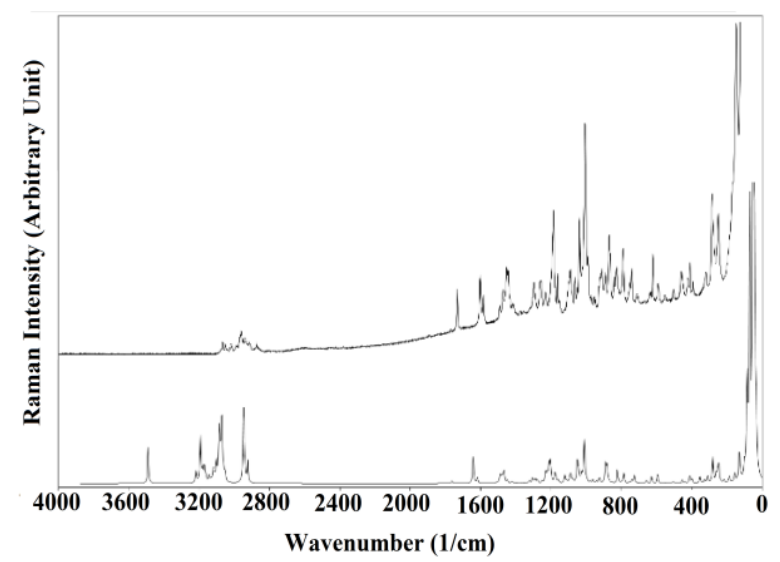

Figure 3. The experimental and simulated Raman spectra of cyclopentolate

\subsection{1. $\mathrm{C}-\mathrm{H}$ and $\mathrm{O}-\mathrm{H}$ vibrations}

The $\mathrm{C}-\mathrm{H}$ stretching vibrations in phenyl ring are between 3100 and $3000 \mathrm{~cm}^{-1}$ (Akyıldırım et al, 2017; Silverstein et al, 2005; Bilkan, 2017; Stuart, 2004; Lambert and Shurvell, 1987; Colthup et al, 1964). The C-H stretching bands in phenyl ring were observed at 3090 and $3105 \mathrm{~cm}^{-1}$ in FT-IR spectrum and at 3068 $\mathrm{cm}^{-1}$ in Laser-Raman spectrum. These bands were computed in the range of $3123-3069 \mathrm{~cm}^{-}$ ${ }^{1}$ in our calculations (Palafox, 2000). The C1$\mathrm{H} 2$ stretching vibration was not observed in FT-IR and Laser-Raman spectra, whereas it was calculated at $2998 \mathrm{~cm}^{-1}$. The HCC inplane bending vibrational modes ( $\delta \mathrm{HCC})$ of phenyl ring were obtained at 1472 (IR)-1474 (R) (exp.)/1470 (cal.), 1429 (cal.), 1307 (IR)1299 (R)/1311 (cal.), 1158 (R) (exp.)/1164 (cal.), 1136 (cal.) and 1067 (cal.) $\mathrm{cm}^{-1}$ as generally combined with $\mathrm{CC}$ stretching vibrations of ring, whereas the HCCC out-ofplane bending vibrations ( $\tau$ HCCC) were found at 967 (IR) (exp.)/967 (cal.), 952 (IR)953 (R) (exp.)/952 (cal.), 912 (R)/913 (cal.), 893 (IR)-890 (R) (exp.)/897 (cal.), 829 (R)/827 (cal.) and 690 (cal.) $\mathrm{cm}^{-1}$ mainly as individual bands (Palafox, 2000).

The O-H stretching band in 1hydroxycyclopentyl group of the title molecule was observed at $3230 \mathrm{~cm}^{-1}$ in FT-IR spectrum, while it was computed at $3386 \mathrm{~cm}^{-}$ 1. The $\mathrm{OH}$ in-plane bending vibrations ( $\delta$ HOC) were found at 1375 (IR)-1372 (R)/1379 (cal. with $34 \%$ contribution of PED) $\mathrm{cm}^{-1}$ and 1386 (cal. with $37 \%$ contribution of PED) $\mathrm{cm}^{-1}$. The $\mathrm{OH}$ out-of-plane bending mode ( $\tau$ HOCC) was not observed in both FTIR and Laser-Raman spectra, whereas it was computed at $682 \mathrm{~cm}^{-1}$ with $72 \%$ contribution of PED. 


\subsubsection{CO vibrations}

The $\mathrm{C}=\mathrm{O}$ stretching band in the carboxyl group gives strong absorption band in the region 1870-1540 $\mathrm{cm}^{-1}$ (Akyıldırım et al, 2017; Silverstein et al, 2005; Bilkan, 2017; Stuart, 2004; Lambert and Shurvell, 1987; Colthup et al, 1964). The $\mathrm{C}=\mathrm{O}$ stretching vibration of cyclopentolate was observed at 1735 (IR)-1734 (R) $\mathrm{cm}^{-1}$ and the computed scaled wavenumber value for this band was obtained at $1710 \mathrm{~cm}^{-1}$. The $\mathrm{C}-\mathrm{O}$ stretching vibrations of cyclopentolate were observed at 991 (IR)-990 (R), 867 (R) and 838 (R) $\mathrm{cm}^{-1}$. The computed values (with $\mathrm{X} \%$ contribution of PED) for this C-O stretching bands $(v \mathrm{C} 16 \mathrm{O} 16, v \mathrm{C} 17 \mathrm{O} 16$ and $v \mathrm{C} 32 \mathrm{O} 45)$ were obtained at 1124 (33\%), 997 (21\%), 986 (13\%), 900 (11\%), 857 (22\%) and 846 (22\%) $\mathrm{cm}^{-1}$.

\subsubsection{C-N vibrations}

Mani et al. assigned C-N stretching absorption at $1169 \mathrm{~cm}^{-1}$ in FT-IR and $1120 \mathrm{~cm}^{-1}$ in FTRaman (Mani et al, 2008). The C-N stretching vibrations were found in the region of 1260$763 \mathrm{~cm}^{-1}$ for cyclopentolate molecule in Table 2. The bands observed as mixed with other vibrational bands at 1186 (IR)-1186 (R), 1034 (IR)-1038 (R), 921 (IR)-920 (R), 750 (IR)-753 (R) $\mathrm{cm}^{-1}$ can be assigned to $\mathrm{CN}$ stretching modes. The computed values for $\mathrm{CN}$ stretching modes in 2-(dimethylamino)ethyl group of the title molecule were obtained at 763 (41\%), 774 (16\%), 934 (35\%), 1020 (43\%), 1044 (29\%), $1188(15 \%)$ and 1260 $(14 \%) \mathrm{cm}^{-1}$. These observed and calculated values coincide with similar structures in the literature (Sroczyński and Malinowski, 2017; Sert et al, 2017).

\subsubsection{C-C vibrations}

The CC stretching vibrations of aromatic rings usually occur at the interval of 1400-1650 $\mathrm{cm}^{-1}$ (Palafox, 2000; Hirschler et al (1994; Angelova et al, 1992; Sroczyński and Malinowski, 2017; Akyıldırım et al, 2017; Silverstein et al, 2005; Bilkan, 2017; Stuart, 2004; Lambert and Shurvell, 1987; Colthup et al, 1964; Mani et al, 2008; Sert et al, 2017; Temel et al, 2015). The $v C C$ stretching bands in this region are called skeletal vibrations of aromatic ring. The $\mathrm{CC}$ bands can also be observed as combined with other vibrations (Akyıldırım et al, 2017) below $1400 \mathrm{~cm}^{-1}$ as given in Table 2. The vibrational bands observed at 1605 (IR)-1604 (R), 1584 (IR and R), 1307 (IR)-1299 (R), 1028 (IR)-1031 (R) $\mathrm{cm}-1$ were assigned to the $\mathrm{CC}$ stretching vibration modes in phenyl ring. The vibrational bands observed at 1101 (IR)-1090 (R), 991 (IR)-990 (R), 929 (R), 750 (IR)-753 (R) and 703 (IR) $\mathrm{cm}^{-1}$ are assigned to the CC stretching vibration in 1-hydroxycyclopentyl ring. The vibrational bands observed at 589 (IR)-592 (R), 621 (R) and 973 (R) $\mathrm{cm}^{-1}$ were assigned to the $\mathrm{CCC}$ in-plane bending vibration modes ( $\delta$ phenyl). Similarly, the CCCC torsional modes in phenyl ring gave rise to absorption bands at $410(\mathrm{R})$ and 501 (IR)-502 (R) cm-1 (Palafox, 2000). The CCC in-plane bending vibrations ( $\delta$ cycl.) in 1hydroxycyclopentyl group were found at 640 (R)/639 (cal. with PED contribution of 29\%), 643 (IR)-644(R)/641 (cal. with PED contribution of $16 \%$ ) and 861 (IR)-870 (R)/865 (cal. with PED contribution of $38 \%$ ) $\mathrm{cm}^{-1}$. The CC stretching ( $\mathrm{vCC}$ ) and CCCC torsional modes ( $\tau$ cycl.) in 1hydroxycyclopentyl group are listed in Table 2.

\subsection{5 $\mathrm{CH}_{3}$ and $\mathrm{CH}_{2}$ vibrations}

The asymmetric and symmetric stretching vibrations of methyl and methylene $\left(\mathrm{CH}_{3}\right.$ and $\mathrm{CH}_{2}$ ) groups were expected to be in the range of 3000-2800 cm $\mathrm{cm}^{-1}$ (Roeges, 1994; 
Ambujakshan et al, 2008; Gökce and Bahceli, 2013). The asymmetric stretching bands can sometimes rise up to $3050 \mathrm{~cm}^{-1}$. The experimental and calculated wavenumbers for scissoring, twisting and rocking modes for methyl and methylene groups are summarized in Table 2. In the present study, $\mathrm{CH}_{3}$ asymmetric stretching appeared at $3039 \mathrm{~cm}-1$ in FT-IR spectra and $3045 \mathrm{~cm}^{-1}$ in LaserRaman spectra. The six asymmetric stretching vibrations in two $\mathrm{CH}_{3}$ groups were computed at 3041, 3029, 2994, 2992, 2988 and 2986 $\mathrm{cm}^{-1}$ with various contributions of PED. The $\mathrm{CH}_{3}$ symmetric stretching bands appeared at 2880 and $2885 \mathrm{~cm}^{-1}$ in FT-IR spectra and at $2872 \mathrm{~cm}^{-1}$ in Laser-Raman spectra and they were calculated at 2854 and $2862 \mathrm{~cm}^{-1}$. The two asymmetric and two symmetric stretching modes for $\mathrm{CH}_{2}$ groups in aliphatic chain were obtained at 3051 (R) (exp.)/3052 (cal.) and 3039 (IR)-3045 (R) (exp.)/3041 (cal. with PED contribution below $10 \%) \mathrm{cm}^{-1}$ and at 2994 (cal.) and at 2990 (IR-R) (exp.)/2992 (cal.) and 2988 (cal.) $\mathrm{cm}^{-1}$, respectively. Similarly, the four asymmetric stretching modes for $\mathrm{CH}_{2}$ groups in cycl. ring were computed at 3028, 3022, 3009 and $2993 \mathrm{~cm}^{-1}$, whereas the symmetric modes for these groups were calculated at 2980, 2972, 2969 and $2962 \mathrm{~cm}^{-1}$. The recorded bands for these asymmetric and symmetric stretching modes of $\mathrm{CH}_{2}$ groups in cycl. ring were observed at 3017 (IR)-3020 (R) $\mathrm{cm}^{-1}$ and at 2964 (IR)2962 (R), 2953 (IR)-2943 (R) and 2927 (IR)2915 (R) $\mathrm{cm}^{-1}$, respectively. The bending modes for $\mathrm{CH}_{3}$ and $\mathrm{CH}_{2}$ groups were observed in the ranges of $1456-1375 \mathrm{~cm}^{-1}$ in IR spectrum and 1454-1372 $\mathrm{cm}^{-1}$ in LaserRaman spectrum, while they were calculated in the range of $1463-1379 \mathrm{~cm}^{-1}$. The scissoring vibrations for $\mathrm{CH}_{3}$ groups were found at 1456 (IR)-1454 (R) (exp.)/1450 (cal.), 1452 (IR-R) (exp.)/1446 (cal.), 1440 (cal.), 1438 (cal), 1435 (cal.) and 1421 (R) (exp.)/1426 (cal.) $\mathrm{cm}^{-1}$, while the symmetric bending modes (or umbrella vibration) were obtained at 1399 (IR) (exp.)/1406 (cal.), 1386 (cal.) and 1375 (IR)-1375 (R) (exp.)/1379 (cal.) $\mathrm{cm}^{-1}$. The four $\mathrm{CH}_{3}$ rocking vibrational modes were found at 1149 (IR) (exp.)/1143 (cal. (48\%)), 1080 (IR) (exp.)/1078 (cal. (66\%)), 1034 (IR)1038(R)/1044 (cal. (18\%)) and 1020 (cal. $(10 \%) \mathrm{cm}^{-1}$. These values are consistent with the experimental FT-IR values at 1149, 1080 and $1034 \mathrm{~cm}^{-1}$ and the Laser-Raman value at $1038 \mathrm{~cm}^{-1}$. The two $\mathrm{CH}_{3}$ torsional vibrational modes were found at 256 and $238 \mathrm{~cm}^{-1}$ by the B3LYP/aug-cc-pVDZ calculation. The scissoring, wagging, twisting and rocking modes for $\mathrm{CH}_{2}$ groups in aliphatic chain and 1-hydroxycyclopentyl were listed in Table 2.

\section{3. ${ }^{1} \mathrm{H}$ and ${ }^{13} \mathrm{C}$ NMR Chemical Shift Analyses}

NMR analysis, used in organic structure determination, is associated with the spin orientation direction. In NMR analysis, chemical shifts are measured in parts per million (ppm). ${ }^{1} \mathrm{H}$ and ${ }^{13} \mathrm{C}$ NMR chemical shift calculated with gauge-including atomic orbital (GIAO) approach using Gaussian09 software shows good agreement with the experimental chemical shift. Figure 4 shows the experimental ${ }^{1} \mathrm{H}$ and ${ }^{13} \mathrm{C}$ NMR chemical shift spectra of cyclopentolate. The experimental ${ }^{1} \mathrm{H}$ and ${ }^{13} \mathrm{C}$ chemical shift values measured in DMSO-d6 solvent and the chemical shift values calculated at the B3LYP/aug-cc-pVDZ level in vacuum and DMSO solvent are shown in Table 3 . The $\mathrm{R}^{2}$ and RMSD values between the experimental and computed ${ }^{13} \mathrm{C}$ NMR chemical shifts were found as 0.99452 and 3.8 ppm, respectively. 


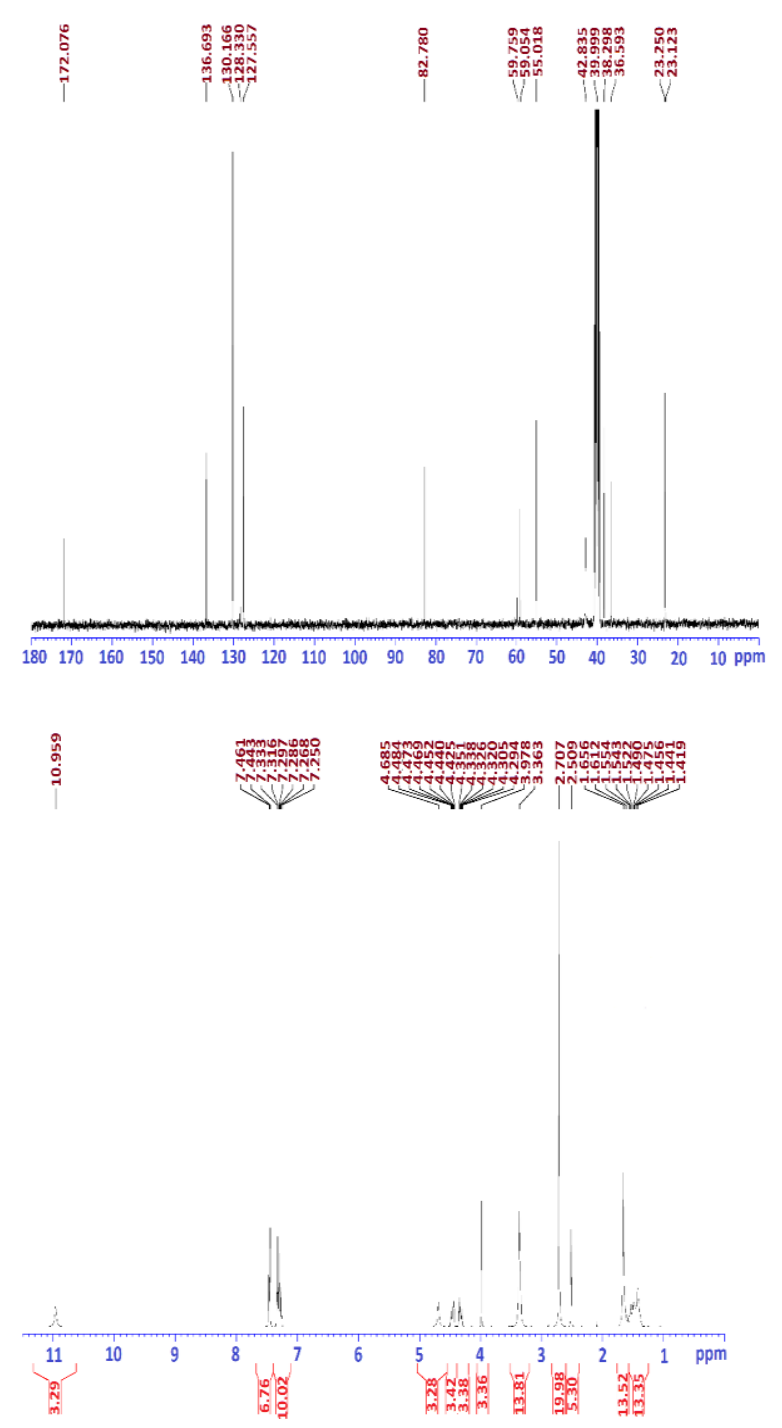

Figure 4. The experimental ${ }^{1} \mathrm{H}$ (bottom) and ${ }^{13} \mathrm{C}$ (top) NMR chemical shift spectra of cyclopentolate

\begin{tabular}{|c|c|c|c|c|c|}
\hline Atom & $\delta$ & $\delta_{1}$ & Atom & $\delta$ & \\
\hline $\mathrm{C} 1$ & 55.018 & 64.600 & $\mathrm{H} 2$ & 3.978 & 4.065 \\
\hline C3 & 136.693 & 139.343 & H7 & \multirow{5}{*}{$\begin{array}{c}7.250- \\
7.461\end{array}$} & 7.573 \\
\hline $\mathrm{C} 4$ & 130.166 & 130.788 & H9 & & 8.468 \\
\hline $\mathrm{C} 5$ & 130.166 & 128.689 & H11 & & 7.681 \\
\hline C6 & 128.330 & 126.450 & H12 & & 7.743 \\
\hline $\mathrm{C} 8$ & 128.330 & 126.204 & $\mathrm{H} 13$ & & 7.685 \\
\hline $\mathrm{C} 10$ & 127.557 & 125.906 & $\mathrm{H} 18$ & \multirow{2}{*}{$\begin{array}{r}4.294- \\
4.484\end{array}$} & 3.754 \\
\hline C14 & 172.076 & 179.336 & H19 & & 5.512 \\
\hline C17 & 59.759 & 60.796 & $\mathrm{H} 21$ & \multirow{2}{*}{3.363} & 2.164 \\
\hline $\mathrm{C} 20$ & 59.054 & 60.353 & $\mathrm{H} 22$ & & 2.961 \\
\hline C24 & 42.835 & 40.243 & $\mathrm{H} 25$ & \multirow{3}{*}{2.707} & 2.264 \\
\hline $\mathrm{C} 28$ & 42.835 & 46.103 & $\mathrm{H} 26$ & & 1.572 \\
\hline C32 & 82.780 & 89.952 & $\mathrm{H} 27$ & & 2.955 \\
\hline C33 & 36.593 & 34.734 & $\mathrm{H} 29$ & \multirow{3}{*}{2.707} & 2.437 \\
\hline C34 & 38.298 & 38.752 & $\mathrm{H} 30$ & & 2.379 \\
\hline C35 & 23.250 & 23.633 & $\mathrm{H} 31$ & & 2.104 \\
\hline C38 & 23.123 & 24.318 & $\mathrm{H} 36$ & \multirow{8}{*}{$\begin{array}{l}1.419- \\
1.656\end{array}$} & 2.040 \\
\hline $\mathbf{R}^{2}$ & \multicolumn{2}{|c|}{0.99452} & $\mathrm{H} 37$ & & 1.535 \\
\hline RMSD & \multicolumn{2}{|c|}{$3.8 \mathrm{ppm}$} & $\mathrm{H} 41$ & & 1.427 \\
\hline & & & $\mathrm{H} 42$ & & 1.033 \\
\hline & & & $\mathrm{H} 43$ & & 1.843 \\
\hline & & & $\mathrm{H} 44$ & & 1.616 \\
\hline & & & H39 & & 1.778 \\
\hline & & & $\mathrm{H} 40$ & & 1.472 \\
\hline & & & $\mathrm{H} 46$ & $\begin{array}{c}4.685 \text { or } \\
10.959\end{array}$ & 5.442 \\
\hline
\end{tabular}

${ }^{1} \mathrm{H}$ chemical shift values were computed at the intervals of 1.033-8.468 ppm in DMSO. The experimental chemical shifts of $1 \mathrm{H}$ are measured in the range of $1.419-10.959 \mathrm{ppm}$. The chemical shift for $\mathrm{H} 2$ is experimentally located at $3.978 \mathrm{ppm}$. The experimental chemical shifts of hydrogen atoms $(\mathrm{H} 7, \mathrm{H} 9$, $\mathrm{H} 11, \mathrm{H} 12, \mathrm{H} 13)$ in phenyl ring are measured in the range of 7.250-7.461 ppm and they were calculated in the region of 7.573-8.468 ppm. The experimental chemical shifts of H18 and $\mathrm{H} 19$ hydrogen atoms are measured in the range of 4.294-4.484 ppm and H18 and H19 hydrogen atoms were calculated at $3.754 \mathrm{ppm}$ and 5.512 ppm, respectively. The experimental chemical shifts of $\mathrm{H} 21$ and $\mathrm{H} 22$ hydrogen atoms were measured as $3.363 \mathrm{ppm}$ and $\mathrm{H} 21$ and $\mathrm{H} 22$ hydrogen atoms were calculated at $2.164 \mathrm{ppm}$ and $2.961 \mathrm{ppm}$, respectively. The experimental chemical shifts of $\mathrm{H} 25, \mathrm{H} 26$ and $\mathrm{H} 27$ hydrogen atoms were measured as $2.707 \mathrm{ppm}$ and H25, $\mathrm{H} 26$ and $\mathrm{H} 27$ hydrogen atoms were calculated at $2.264 \mathrm{ppm}, 1.572 \mathrm{ppm}$ and $2.955 \mathrm{ppm}$, 
respectively. The experimental chemical shifts of H29, H30 and H31 hydrogen atoms were measured as 2.707 ppm and H29, H30 and H31 hydrogen atoms were calculated at $2.437 \mathrm{ppm}$, $2.379 \mathrm{ppm}$ and $2.104 \mathrm{ppm}$, respectively. The experimental chemical shifts of hydrogen atoms (H36, H37, H41, H42, H43, H44, H39, $\mathrm{H} 40)$ were measured in the range of 1.419$1.656 \mathrm{ppm}$ and $\mathrm{H} 36, \mathrm{H} 37, \mathrm{H} 41, \mathrm{H} 42, \mathrm{H} 43, \mathrm{H} 44$, $\mathrm{H} 39, \mathrm{H} 40$ atoms were calculated at $2.040 \mathrm{ppm}$, 1.535 ppm, 1.427 ppm, 1.033 ppm, 1.843 ppm, $1.616 \mathrm{ppm}, 1.778 \mathrm{ppm}$ and $1.472 \mathrm{ppm}$, respectively. The largest deviation between the calculated and experimental 1H NMR chemical shifts ( $\delta$ exp.- $\delta$ cal.) was observed for H46. The weakest interaction was observed for $\mathrm{H} 46$ with $4.685 \mathrm{ppm}$ and the strongest interaction was observed for $\mathrm{H} 46$ with $10.959 \mathrm{ppm}$ in experimental ${ }^{1} \mathrm{H}$ chemical shift spectra. The experimental NMR chemical shifts of protons in phenyl and dimethylamino groups of cyclopentolate molecule were recorded at the interval of $\sim 7.38-7.50 \mathrm{ppm}$ and at $\sim 2.8 \mathrm{ppm}$ by Roy and Guillory (1996) respectively. These values are in a good agreement with the recorded and computed data in our study.

The ${ }^{13} \mathrm{C}$ chemical shifts were calculated in the range of 24.318-179.336 in DMSO ppm, while they were experimentally recorded in the range of 23.123-172.076 ppm. The largest deviation between the calculated and experimental ${ }^{13} \mathrm{C}$

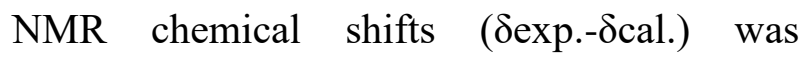
obtained for $\mathrm{C} 1$ with $9.582 \mathrm{ppm}$, whereas the smallest deviation was found for C35 with $0.383 \mathrm{ppm}$. The NMR chemical shift value for C14 carbon atom in ester group of cylopentolate was obtained at 172.076 (exp)/179.336 (cal.) ppm, due to attached to electronegative oxygen atoms. Likewise, the C32 carbon atom bonded to $\mathrm{O} 45$ atom was given at 82.780 (exp.)/89.952 (cal.) ppm NMR signal. The NMR signals for aromatic sp2 carbons were recorded at the interval of
127.557-136.693 ppm, whereas the sp3 carbon atoms in aliphatic and 1-hydroxycyclopentyl groups were observed in the region of 23.123$59.759 \mathrm{ppm}$.

\subsection{UV-Vis. Analysis}

The UV-Vis. spectrum of cyclopentolate dissolved in methanol was recorded in the region of 200-600 $\mathrm{nm}$. UV-Vis. calculation was performed using TD-DFT method in methanol. The simulated and measured UVVis. electronic absorption spectra are given in Figure 5. Additionally, the experimental and computed electronic absorption wavelengths, electronic transitions, oscillator strengths, excitation energies and major contributions are listed Table 5. The major contributions for the computed electronic wavelengths were obtained by GaussSum 3.0 program (O'boyle et al, 2008).

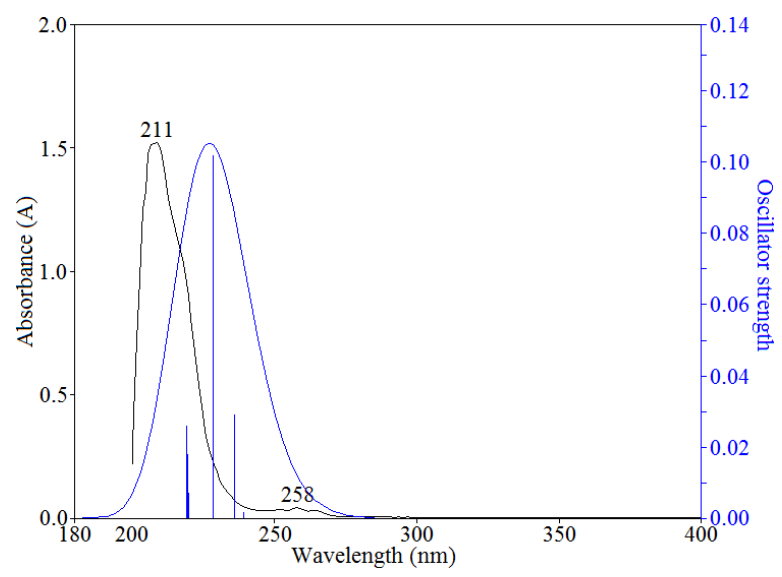

Figure 5. The recorded (black) and simulated (blue) UV-Vis. spectra in methanol of cyclopentolate

The wavelength recorded at $258 \mathrm{~nm}$ in experimental UV-Vis. spectrum can be assigned to $n \rightarrow \pi^{*}$ transition. This transition can be ascribed from dimetilamino group to phenyl ring in cyclopentolate molecule. The calculated wavelength corresponding to this experimental value was obtained at $235.78 \mathrm{~nm}$ with 0.0291 values of oscillator strength. The 
major contribution for these computed values were found as $\mathrm{H} \rightarrow \mathrm{L}(85 \%)$. Similarly, the wavelength recorded at $211 \mathrm{~nm}$ corresponds to $\pi \rightarrow \pi^{*}$ electronic transition that can be attributed to the phenyl ring. The computed wavelengths and oscillator strength values were found as $228.29 \mathrm{~nm}$ and 0.1017 (the band with the highest intensity in the computed UV-Vis. wavelengths), respectively. The major contributions computed for this computed value were obtained as $\mathrm{H}-1 \rightarrow \mathrm{L}(70 \%)$ and $\mathrm{H}-1 \rightarrow \mathrm{L}+3$ $(10 \%)$. The other computed wavelengths and their major contributions were listed in Table 4. The experimental and computed wavelengths and electronic transitions are in good harmony (Szwed et al, 2013).

\begin{tabular}{l}
\hline \multicolumn{6}{|l|}{ Table 4. The experimental and computed UV-Vis. } \\
parameters and electronic transitions in methanol of \\
cyclopentolate. \\
\hline $\begin{array}{l}\text { The } \\
\text { experimental }\end{array}$
\end{tabular}

\subsection{HOMO-LUMO Analyses}

The highest and the lowest occupied molecular orbitals (HOMO and LUMO) are also referred to as the frontier molecule orbitals (FMOs) (Fukui, 1982). The HOMOs and LUMOs are donor and acceptor groups filled and unfilled by electrons, respectively (Buyukuslu et al, 2010). The simulated
HOMO and LUMO surfaces, energy values and their shapes for the title molecule are given in Figure 6. The calculated HOMO-1, HOMO, LUMO and LUMO+1 energy values are $-6.8339 \mathrm{eV},-6.6306 \mathrm{eV},-0.7614 \mathrm{eV}$ and $0.5714 \mathrm{eV}$, respectively at the DFT/B3LYP/aug-cc-pVDZ level. The energy gap between HOMO and LUMO is computed at $5.8692 \mathrm{eV}$. The energy gap between HOMO-1 and LUMO+1 is computed at $6.2625 \mathrm{eV}$. The HOMO is localized on the dimetilamino group excluding phenyl ring and 1-hydroxycyclopentyl group, while the LUMO is placed on overall molecular structure except (dimethylamino)-ethyl and 1hydroxycyclopentyl groups. The HOMO and LUMO localizations on cyclopentolate are formed by $\mathrm{n}$ molecular orbitals on nitrogen atom and $\pi^{*}$ molecular orbitals of aromatic ring, respectively. Similarly, the HOMO-1 is mainly localized on the bonding $\pi$ molecular orbitals of aromatic ring, whereas the LUMO+1 is placed on anti-bonding $\pi^{*}$ molecular orbitals of phenyl ring. As a result, the $n \rightarrow \pi^{*}$ electronic transition can be associated with $\mathrm{HOMO} \rightarrow$ LUMO and $\mathrm{HOMO} \rightarrow \mathrm{LUMO}+1$, whereas the $\pi \rightarrow \pi^{*}$ electronic transition can be assigned to HOMO- $\rightarrow$ LUMO and HOMO$1 \rightarrow$ LUMO +1 . These notations can be confirmed by electronic transitions observed and assigned in UV-Vis. spectrum of cyclopentolate.

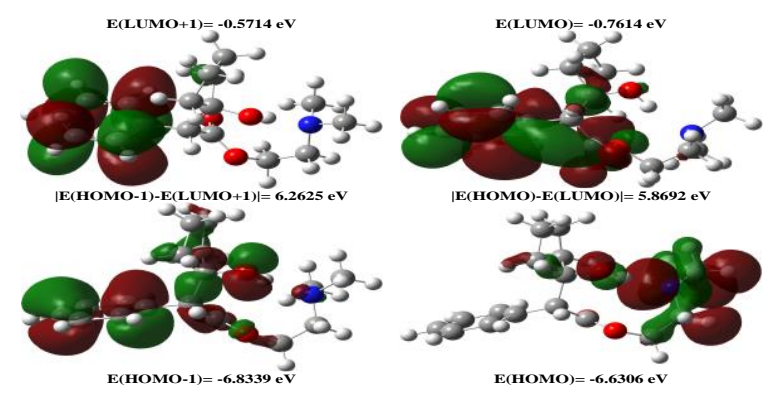

Figure 6. The HOMO-1, HOMO, LUMO and LUMO+1 plots and their energy values of cyclopentolate 


\section{Conclusion}

In this research, conformational analysis was carried out to obtain the ground stable molecular structure with the minimum energy of cyclopentolate. As a result of conformational analysis, four molecular geometric forms in at the lowest energies were optimized with the B3LYP/aug-cc-pVDZ level. The computed molecular energy for the most stable conformer I was obtained as -943.40481341 Hartrees. By considering conformer I, the structural, spectroscopic (IR, Raman, NMR and UV-Vis.) and HOMOLUMO analyses for cyclopentolate were performed using theoretical computational methods. The computed spectral properties were compared with the experimental data. A weak intra-molecular hydrogen bond interaction in cyclopentolate molecule was observed in $\quad \mathrm{O} 45-\mathrm{H} 46 \ldots \mathrm{N} 23$ $(\mathrm{r}(\mathrm{H} 46 \ldots \mathrm{N} 23)=2.027 \quad \AA \quad$ and $\mathrm{A}(\mathrm{O} 45-$ $\left.\mathrm{H} 46 \ldots \mathrm{N} 23)=164.1^{\circ}\right)$. Due to the influence of this interaction, the $\mathrm{OH}$ stretching band was observed at $3230 \mathrm{~cm}^{-1}$. The electronic transitions in UV-Vis. spectra supported with charge transfers in the HOMO and LUMO analyses of the molecule were determined as $\pi \rightarrow \pi^{*}(211 \mathrm{~nm})$ and $\mathrm{n} \rightarrow \pi^{*}(258 \mathrm{~nm})$.

\section{References}

Akyıldırım, O., Gökce, H., Bahçeli, S. and Yüksek, H. (2017). "Theoretical and spectroscopic (FT-IR, NMR and UV-Vis.) characterizations of 3-p-chlorobenzyl-4-(4carboxybenzy lidenamino)-4,5-dihydro- $1 \mathrm{H}$ 1,2,4-triazol-5-one molecule", Journal of Molecular Structure, 1127, 114-123.

Ambujakshan, K. R., Madhavan, V.S., Varghese, H.T., Panicker, C. Y., TemizArpaci, O., Tekiner-Gulbas B. and Yildiz I.
(2008). "Vibrational spectroscopic studies and ab initio calculations of 5-methyl-2-(pmethylaminophenyl)benzoxazole, Spectrochim". Spectrochimica Acta Part A, 69, 782-788.

Angelova, O., Macicek, J., Vassilev, N.G., Momchilova, S. and Petrova, J. (1992). "Structures of threo (RR,SS) diethyl ester of 2-hydroxy-1,2-diphenylethylphosphonic acid and $( \pm)$ diethyl ester of (1hydroxycyclopentyl)(2-methylphenyl) methylphosphonic acid", J. Crystallogr. Spectrosc. Res., 22, 253-258.

Becke, A.D. (1993). "Density-functional thermochemistry. III. The role of exact Exchange", J. Chem. Phys. 98, 5648-5652.

Bilkan, M. T. (2017). "Structural and spectroscopic studies on dimerization and solvent-ligand complexes of Theobromine". Journal of Molecular Liquids, 238, 523-532.

Colthup, N.B., Daly, L.H. and Wiberley, E. (1964). Introduction to Infrared and Raman Spectroscopy, Academic Press, New York.

Dennington, R., Keith, T. and Millam, J. (2009). GaussView, Version 5, Semichem Inc., Shawnee Mission KS.

Ditchfield, R. (1974). "Self-consistent perturbation theory of diamagnetism. I. A gauge-invariant LCAO method for N.M.R. chemical shifts", Molecular Physics, 27, 789807.

Farhood, Q. K. (2012). "Cycloplegic Refraction in Children with Cyclopentolate versus Atropine“, Journal of Clinical and Experimental Ophthalmol., 3(7), 1-5. 
Frag, E. Y. Z., Mohamed, G.G., El-Dien F.A.N., and Mohamed, M. El-Badry. (2011). "The Use of Integrated Analytical Tools for Determination of Cyclopentolate and Naphazoline Hydrochlolrides in Pure and Pharmaceutical Preparations", Pharmaceutica Analytica Acta, 2(1), 1-6.

Frisch, M.J. et al. (2009). Gaussian 09, Revision C.01, Gaussian, Inc., Wallingford CT.

Fukui, K. (1982). "Role of frontier orbitals in chemical reactions", Science, 218, 747-754.

H. Buyukuslu, M. Akdogan, G. Yildirim and C. Parlak. (2010). "Ab initio Hartree-Fock and density functional theory study on characterization of 3-(5-methylthiazol-2yldiazenyl)-2-phenyl-1H-indole",

Spectrochim. Acta Part A, 75, 1362-1369.

H. Gökce and S. Bahçeli. (2013). "The molecular structures, vibrational spectroscopies (FT-IR and Raman) and quantum chemical calculations of nalkyltrimethylammonium bromides". Opt. Spectrosc, 115, 632-644.

Hirschler, J., Berger, B. and Bolte, M. (1994). "A dimeric (phenylsulfonyl) oxazolidine", Acta Crystallographica, C50, 1279-1281.

Hung, K. C., Huang, H.M. and Lin, P.W. (2015). "Changes of intraocular pressure and refractive status in children following cycloplegic refraction with $1 \%$ cyclopentolate and $1 \%$ tropicamide", Taiwan Journal of. Ophthalmology, 5, 124-127.

Izake, E.L. (2007). "Chiral discrimination and enantioselective analysis of drugs: An overview", Journal of Pharmaceutical Sciences, 96, 1659-1676.
Jamr'oz, M.H. (2004). Vibrational Energy Distribution Analysis VEDA4, Warsaw.

Lambert, J. B., Shurvell, H. F., and Cooks, R. G. (1987). Introduction to Organic Spectroscopy, Macmillan Publish, New York, USA.

Lee, C., Yang, W. and Parr, R.G. (1988). "Development of the Colle-Salvetti correlation-energy formula into a functional of the electron density", Phys. Rev. B, , 37, 785-789.

Li, T., Zhou, X., Chen, Z. and Zhou, X. (2015). "Effects of Cyclopentolate on Form Deprivation Myopia in Guinea Pigs", Open Journal of Ophthalmology, 5, 10-18.

London, F. (1937). "Théorie quantique des courants interatomiques dans les combinaisons aromatiques", J. Phys. Radium, 8, 397-409.

Mani, P., Umamaheswari, H., Joshua, B. D. and Sundaraganesan, N. (2008). "Molecular structure, vibrational spectra and NBO analysis of phenylisothiocyanate by density functional method", Journal of Molecular Structure: THEOCHEM, 863, 44-49.

Mericko, D., Lehotay, J. and Cizmárik, J. (2007). "HPLC separation of enantiomers using chiral stationary phases", Čes. slov. Farm, 56(3), 107-113.

Miertus, S., Scrocco, E. and Tomasi, J. (1981). "Electrostatic interaction of a solute with a continuum. Electrostatic interaction of a solute with a continuum. A direct utilization of $\mathrm{AB}$ initio molecular potentials for the prevision of solvent effects", Chemical Physics, 55, 117-129. 
Mindel, J. S. (1994). “Duane's Foundations of Clinical Ophthalmology”, Vol. 3, JB Lippincott, Philadelphia.

O'boyle, M., Tenderholt, A. L. and Langner, K. M. (2008). "cclib: a library for packageindependent computational chemistry algorithms", Journal of Computational Chemistry, 29, 839-845.

Özer, A. (2005). “Görme Optiği ve Refraksiyon”, Tüm Optik ve Optometrik Meslekler Birliği Derneği.

Palafox, M. A. (2000). "Scaling Factors for the Prediction of Vibrational Spectra. I. Benzene Molecule", International Journal of Quantum Chemistry, 77, 661-684.

Roeges, N. P. G. (1994). A Guide to the Complete Interpretation of Infrared Spectral of Organic Structures, John Wiley, Chichester.

Roy, A. K. and Guillory, J.K. (1995). "The kinetics and mechanism of the hydrolysis of cyclopentolate hydrochloride in alkaline solutions", International Journal of Pharmaceutics, 120, 169-178.

Roy. A. K. and Guillory, J. K. (1996). "The effect of cyclodextrins on the aqueous stability of cyclopentolate hydrochloride", International Journal of Pharmaceutics, 138, 37-43.

Runge, E. and Gross, E. K. U. (1984). "Gross, Density-functional theory for time-dependent systems", Physical Review Letter, 52, 9971000.

Russell D. Johnson III (Ed.). (2016). NIST Computational Chemistry Comparison and
Benchmark Database, NIST Standard Reference Database, Number 101, Release 18, October, http://cccbdb.nist.gov/.

Sert, Y., Öztürk, N., Al-Omary, F. A. M., Alaşalvar, C., Al-Shehri, M. M., El-Emam, A. A. and Gökce, H. (2017). "Experimental (FTIR, Laser-Raman and NMR) and theoretical spectroscopic analysis of $3-[(\mathrm{N}-$ methylanilino) methyl]-5-(thiophen-2-yl)1,3,4-oxadiazole-2(3H) -thione", Computational and Theoretical Chemistry, 16(3) 1750024.

Silverstein, R. M., Webster, F. X. and Kiemle, D. J. (2005). Spectroscopic Identification of Organic Compound, 7th ed., John Wiley \& Sons, Inc., USA.

Sroczyński, D. and Malinowski, Z. (2017). "Spectroscopic investigations (FT-IR, UV, $1 \mathrm{H}$ and 13C NMR) and DFT/TD-DFT calculations of potential analgesic drug 2-[2(dimethylamino)ethyl]-6-methoxy-4(pyridin-2-yl)-1(2H)-phthalazinone", Journal of Molecular Structure, 1150, 614-628.

Stuart, B. H. (2004). Infrared Spectroscopy: Fundamentals and Applications, JohnWilley \& Sons, England.

Szwed, K., Gorecki, M. and Frelek, J. (2013). "Enantioselective extraction system containing binary chiral selectors and chromatographic enantioseparation method for determination of the absolute configuration of enantiomers of cyclopentolate", Chromatographia, 76, 16031611.

Temel, E., Alaşalvar, C., Gökçe, H., Güder, A., Albayrak, Ç., Alpaslan, Y. B., Alpaslan, G. and Dilek, N. (2015). "DFT calculations, spectroscopy and antioxidant activity studies 
on

(E)-2-nitro-4-

[(phenylimino)methyl]phenol",

Spectrochimica Acta A, 136, 534-546.

Trotter, J. (1960). "Bond lengths in benzene derivatives: Hybridization or resonance", Tetrahedron, 8, 13-22.

Vale, J. and Cox, B. (1978). "Drugs and the Eye”, Butterworth \& Co (Publishers) Ltd.

Wolinski, K., Hinton, J.F. and Pulay, P. (1990). "Efficient implementation of the gauge-independent atomic orbital method for NMR chemical shift calculations", Journal of the American Chemical Society, 112, 82518260 . 